Research Article

\title{
A New TOPSIS Approach Using Cosine Similarity Measures and Cubic Bipolar Fuzzy Information for Sustainable Plastic Recycling Process
}

\author{
Muhammad Riaz $\mathbb{D D}^{1}{ }^{1}$ Dragan Pamucar ${ }^{(D)},{ }^{2}$ Anam Habib, ${ }^{1}$ and Mishal Riaz ${ }^{1}$ \\ ${ }^{1}$ Department of Mathematics, University of the Punjab, Lahore, Pakistan \\ ${ }^{2}$ Department of Logistics, Military Academy, University of Defence in Belgarde, Belgarde 11000, Serbia \\ Correspondence should be addressed to Dragan Pamucar; dragan.pamucar@va.mod.gov.rs
}

Received 9 September 2021; Accepted 30 October 2021; Published 15 December 2021

Academic Editor: Ali Ahmadian

Copyright (c) 2021 Muhammad Riaz et al. This is an open access article distributed under the Creative Commons Attribution License, which permits unrestricted use, distribution, and reproduction in any medium, provided the original work is properly cited.

A cubic bipolar fuzzy set (CBFS) is a robust paradigm to express bipolarity and vagueness in terms of bipolar fuzzy numbers and interval-valued bipolar fuzzy numbers. The abstraction of similarity measures (SMs) has a large number of applications in various fields. Therefore, in this study, taking the advantage of CBFSs, three cosine similarity measures for CBFSs are proposed successively by using cosine of the angle between two vectors, new distance measures, and cosine function. Some key properties of these similarity measures (SMs) are explored. Based on suggested SMs, the problem of bacteria recognition is analyzed and an important application is provided to exhibit the efficiency of proposed SMs for CBF information. Moreover, the TOPSIS approach based on cosine SMs is developed for multicriteria group decision-making (MCGDM) problems. An illustrative example about the selection of sustainable plastic recycling process is presented to discuss the efficiency of the suggested MCGDM technique.

\section{Introduction}

Fuzzy set (FS) theory [1] by using the concept of membership function (MF) is a robust approach for modeling uncertainty. A membership function is the generalization of characteristic function in the crisp set theory. An intervalvalued fuzzy set (IVFS) [2] is the generalization of FS that assigns an interval of membership grades to the elements in the universe. The idea of orthopair has been extended to the ordered pair of membership grade (MG) and nonmembership grade (NMG) in the studies of intuitionistic fuzzy sets (IFSs) [3], Pythagorean fuzzy sets (PFSs) [4, 5], and q-rung orthopair fuzzy sets (q-ROPFSs) [6]. The values of MG and NMG are the elements of $[0,1]$, i.e., any real number between 0 and 1 . A number $(\mu, \nu)$ is called an intuitionistic fuzzy number (IFN) if $0 \leq \mu+\nu \leq 1$, a Pythagorean fuzzy number (PFN) if $0 \leq \mu^{2}+\nu^{2} \leq 1$, and a $q$-rung orthopair fuzzy number (q-ROFN) if $0 \leq \mu^{q}+\nu^{q} \leq 1$, $(q \geq 1)$.

In many real-life problems, the indeterminacy is an essential factor to express expert opinion of the decision makers (DMs). To express such information, the idea of ordered triples with three components (MG, indeterminacy, and NMG) of neutrosophic set (NS) [7] and single-valued neutrosophic set (SVNS) [8] has been focused by many researchers. The concepts of spherical fuzzy sets [9-11] and picture fuzzy sets $[12,13]$ are strong models to deal with uncertain real-life problems with three components.

Zhang $[14,15]$ proposed the notion of bipolar fuzzy set (BFS) and bipolar (crisp) set to deal with bipolarity and fuzziness. Lee [16] proposed some results for bipolar-valued fuzzy sets. Deli et al. [17] studied bipolar neutrosophic set (BNS) and proposed novel features of BNSs with application towards MCDM. Wei et al. [18] studied interval-valued 
bipolar fuzzy set (IVBFS) for uncertainty and bipolarity and positive and negative intervals based MCDM approach.

A hybrid concept of cubic set (CS) has been studied by Jun et al. [19]. He proposed novel concepts of internal (external) cubic sets of $P$-intersection, $P$-union, $R$-intersection, and $R$-union. The degree of similarity between two objects can be determined by the notion of similarity measure (SM). Ye [20] introduced cosine similarity measures for IFSs. Wei and Wei [21] defined 10 different kinds of similarity measures for medical science and pattern recognition using PFS information using hesitation, MG and NMG, cosine function, and distance measures. Ulucay et al. [22] proposed new SMs for bipolar neutrosophic sets (BNSs) like hybrid vector SMs, Dice SMs, weighted Dice SMs, and weighted hybrid vector SMs. A comparative analysis for different values of the operational parameter is developed to express the validity of suggested SMs. Abdel-Basset et al. [23] investigated medical diagnosis of bipolar disorders by using BNSs-based SMs. They developed new MADM methods based on SMs and their weighted versions and illustrated them with some numerical examples. Tu et al. [24] suggested Dice SMs and Jaccard and cotangent SMs for neutrosophic cubic sets (NCSs) and applied them in MCDM. Lu and Ye [25] defined cosine SMs for NCSs by using cosine functions, distance, and cosine angle of two vectors. They investigated certain properties and propositions of proposed SMs. Peng et al. $[26,27]$ studied information measures for PFSs and q-ROFSs with corresponding applications in MCDM. Naeem et al. [28] investigated new SMs for PFS information for the analysis of psychological disorder under uncertainty. Hussian and Yang [29] introduced Pythagorean fuzzy Hausdorff metric-based new distance and similarity measures and TOPSIS approach for MCDM.

TOPSIS is a well-known MCDM approach which was first introduced by Hwang and Yoon [30]. Zhang and Xu [31] initiated the Pythagorean fuzzy TOPSIS technique by defining a distance measure. Rani et al. [32] established the TOPIS method based on SMs for the PF environment and applied it for project delivery system selection. Akram et al. [33] developed bipolar fuzzy TOPSIS and utilized it in medical diagnosis. Garg and Arora [34] introduced the IFSSTOPSIS method by using the correlation coefficient for solving MCDM problems. Garg and Kaur [35] developed TOPSIS based on cubic intuitionistic fuzzy (CIFS) information. They proposed a nonlinear-programming-based MCDM approach to deal with cubic intuitionistic fuzzy (CIFS) uncertain information. Riaz and Tehrim [36-38] initiated the novel hybrid model, namely, cubic bipolar fuzzy set (CBFS), by incorporating the features of BFS and IVBFS. They suggested some AOs named as CBF weighted averaging (geometric) AOs with $\mathrm{R}(\mathrm{P})$ orders for external (internal) CBF information.

Ali et al. [39] proposed Einstein geometric aggregation operators using novel complex interval-valued Pythagorean fuzzy sets. Alosta et al. [40] developed a new AHP-RAFSI approach for resolving a location selection problem. Hashemkhani Zolfani et al. [41] introduced a VIKOR- and TOPSIS-focused reanalysis of the MADM methods based on logarithmic normalization. Ramakrishnan and Chakraborty
[42] proposed a cloud TOPSIS model for green supplier selection. Dobrosavljevic and Urosevic [43] suggested analysis of business process management defining and structuring activities. Yorulmaz et al. [44] proposed a robust Mahalanobis distance-based TOPSIS to evaluate the economic development of provinces. Petrovic and Kankaras [45] developed a hybridized IT2FS-DEMATEL-AHPTOPSIS multicriteria decision-making approach as a case study of selection and evaluation of criteria for determination of air traffic control radar position. Badi and Pamucar [46] introduced a supplier selection method for steel-making companies by using combined Grey-MARCOS. Riaz et al. [47] proposed essential characteristics for soft multiset topology and robust MCDM applications.

The advantages and objectives of this manuscript are as follows: (1) To deal with vagueness and bipolarity with cubic bipolar fuzzy sets (CBFSs) which are a superior model to existing bipolar fuzzy models. (2) To define cosine SMs between CBFSs based on cosine of the angle between two vectors, new distance measures, and cosine function. Moreover, their weighted extensions are also introduced. (3) To apply these similarity measures to bacteria recognition problem. (4) To propose the TOPSIS approach based on cosine SMs to deal with the plastic recycling method selection problem.

The arrangement of this manuscript is planned as follows: In Section 2, we discuss some rudimentary concepts of bipolarity and fuzziness. In Section 3, we define cosine SMs, weighted cosine SMs, and related propositions. In Section 4, we establish an algorithm to handle pattern recognition problems under the CBF environment and a complex pattern recognition problem is presented to exhibit the efficiency of proposed algorithm. In Section 5, we introduce TOPSIS approach based on cosine SMs and an application concerning the selection of most sustainable plastic recycling process is discussed. Finally, we assess the validity and usefulness of our suggested technique by comparing it with some existing methodologies. Section 6 is designed for concluding remarks to express advantages and objectives of this manuscript.

\section{Preliminaries}

Some rudimentary concepts can be reviewed to understand the necessary fundamentals related to this manuscript (see $[1,2,14,18,19,36-38])$.

Definition 1 (see [37]). A cubic bipolar fuzzy set (CBFS) $\mathfrak{U}$ on the universe of discourse $\mathfrak{R}$ can be defined as

$$
\mathcal{U}=\{\langle\widetilde{\varrho}, \mathfrak{I}(\widetilde{\varrho}), \mathfrak{S}(\widetilde{\varrho})\rangle: \quad \widetilde{\varrho} \in \mathfrak{R}\},
$$

where $\mathfrak{T}$ is an IVBFS and $\mathfrak{S}$ is a BFS on $\mathfrak{R}$. Thus, CBFS can also be written as

$$
\begin{aligned}
\mathfrak{U}= & \left\{\left\langle\widetilde{\varrho},\left[\rho_{\ell \mathfrak{U}}^{+}(\widetilde{\varrho}), \rho_{\mathfrak{u} \mathfrak{U}}^{+}(\widetilde{\varrho})\right],\left[\rho_{\ell \mathfrak{U}}^{-}(\widetilde{\varrho}), \rho_{\mathfrak{u} \mathfrak{U}}^{-}(\widetilde{\varrho})\right],\right.\right. \\
& \left.\left.\cdot\left(\rho_{\mathfrak{U}}^{+}(\widetilde{\varrho}), \rho_{\mathfrak{U}}^{-}(\widetilde{\varrho})\right)\right\rangle: \quad \widetilde{\varrho} \in \mathfrak{R}\right\},
\end{aligned}
$$

where $\left[\rho_{\ell \mathfrak{U}}^{+}(\widetilde{\varrho}), \rho_{u \mathfrak{U}}^{+}(\widetilde{\varrho})\right] \in I([0,1])$ and $\left[\rho_{\ell \mathfrak{U}}^{-}(\widetilde{\varrho}), \rho_{u \mathfrak{U}}^{-}(\widetilde{\varrho})\right] \epsilon$ $I^{*}([-1,0])$ represent the interval-valued positive and 
negative MGs, respectively, and $\rho_{\mathfrak{U}}^{+}(\widetilde{\varrho}) \in[0,1]$ and $\rho_{\mathfrak{U}}^{-}(\widetilde{\varrho}) \in[-1,0]$ represent the single-valued positive and negative MGs, respectively, of an object $\widetilde{\varrho} \in \mathfrak{R}$.

\subsection{Operations on CBFSs}

Definition 2 (see [37]). Let $\mathfrak{U}=\left\{\left\langle\widetilde{\varrho},\left[\rho_{\ell \mathfrak{U}}^{+}(\widetilde{\varrho}), \rho_{\mathfrak{u} \mathfrak{U}}^{+}(\widetilde{\varrho})\right]\right.\right.$, $\left.\left.\left[\rho_{\ell \mathfrak{U}}^{-}(\widetilde{\varrho}), \rho_{u \mathfrak{U}}^{-}(\widetilde{\varrho})\right],\left(\rho_{\mathfrak{U}}^{+}(\widetilde{\varrho}), \rho_{\mathfrak{U}}^{-}(\widetilde{\varrho})\right)\right\rangle: \widetilde{\varrho} \in \mathfrak{R}\right\}$ and $\mathfrak{Q}=\{\langle\widetilde{\varrho}$, $\left.\left[\rho_{\ell \mathfrak{Q}}^{+}(\widetilde{\varrho}), \quad \rho_{u \mathfrak{Q}}^{+}(\widetilde{\varrho})\right],\left[\rho_{\ell \mathfrak{Q}}^{-}(\widetilde{\varrho}), \rho_{u \mathfrak{Q}}^{-}(\widetilde{\varrho})\right], \quad\left(\rho_{\mathfrak{Q}}^{+}(\widetilde{\varrho}), \rho_{\mathfrak{Q}}^{-}(\widetilde{\varrho})\right)\right\rangle:$ $\widetilde{\varrho} \in \mathfrak{R}\}$ be two CBFSs on $\mathfrak{R}$ and $\lambda>0$. Then, the operations on these CBFSs under $P$-order are given as follows:

(i) $\mathfrak{U} \cup_{P} \mathfrak{Q}=\left\{\left\langle\widetilde{\varrho},\left[\max \left\{\rho_{\ell \mathfrak{U}}^{+}(\widetilde{\varrho}), \quad \rho_{\ell \mathfrak{Q}}^{+}(\widetilde{\varrho})\right\}, \quad \max \left\{\rho_{u \mathfrak{U}}^{+}\right.\right.\right.\right.$ $\left.\left.(\widetilde{\varrho}), \rho_{\mathcal{u Q}}^{+}(\widetilde{\varrho})\right\}\right],\left[\min \left\{\rho_{\ell \mathfrak{U}}^{-}(\widetilde{\varrho}), \rho_{\ell \mathfrak{Q}}^{-}(\widetilde{\varrho})\right\}, \min \left\{\rho_{u \mathfrak{U}}^{-}(\widetilde{\varrho})\right.\right.$, $\left.\left.\rho_{u \mathfrak{Q}}^{-}(\widetilde{\varrho})\right\}\right], \quad\left(\max \left\{\rho_{\mathfrak{U}}^{+}(\widetilde{\varrho}), \rho_{\mathfrak{Q}}^{+}(\widetilde{\varrho})\right\}, \quad \min \left\{\rho_{\mathfrak{U}}^{-}(\widetilde{\varrho}), \rho_{\mathfrak{Q}}^{-}\right.\right.$ $(\widetilde{\varrho})\})\rangle: \widetilde{\varrho} \in \Re\}$

(ii) $\mathfrak{U} \cap_{P} \mathfrak{Q}=\left\{\left\langle\widetilde{\varrho},\left[\min \left\{\rho_{\ell \mathfrak{U}}^{+}(\widetilde{\varrho}), \rho_{\ell \mathfrak{Q}}^{+}(\widetilde{\varrho})\right\}, \quad \min \left\{\rho_{u \mathfrak{U}}^{+}\right.\right.\right.\right.$ $\left.\left.(\widetilde{\varrho}), \rho_{u \mathfrak{Q}}^{+}(\widetilde{\varrho})\right\}\right], \quad\left[\max \left\{\rho_{\ell \mathfrak{U}}^{-}(\widetilde{\varrho}), \rho_{\ell \mathfrak{Q}}^{-}(\widetilde{\varrho})\right\}, \max \left\{\rho_{u \mathfrak{U}}^{-}\right.\right.$ $\left.\left.(\widetilde{\varrho}), \rho_{u \mathfrak{Q}}^{-}(\widetilde{\varrho})\right\}\right],\left(\min \left\{\rho_{\mathfrak{U}}^{+}(\widetilde{\varrho}), \rho_{\mathfrak{Q}}^{+}(\widetilde{\varrho})\right\}, \quad \max \left\{\rho_{\mathfrak{U}}^{-}(\widetilde{\varrho})\right.\right.$, $\left.\left.\left.\left.\rho_{\mathfrak{Q}}^{-}(\widetilde{\varrho})\right\}\right)\right\rangle: \widetilde{\varrho} \in \mathfrak{R}\right\}$

(iii) $\mathfrak{U} \oplus_{P} \mathfrak{Q}=\left\{\left\langle\widetilde{\varrho}, \quad\left[\rho_{\ell \mathfrak{U}}^{+}(\widetilde{\varrho})+\rho_{\ell \mathfrak{Q}}^{+}(\widetilde{\varrho})-\rho_{\ell \mathfrak{U}}^{+}(\widetilde{\varrho}) \rho_{\ell \mathfrak{Q}}^{+}(\widetilde{\varrho})\right.\right.\right.$, $\left.\rho_{u \mathfrak{U}}^{+}(\widetilde{\varrho})+\rho_{u \mathfrak{Q}}^{+}(\widetilde{\varrho})-\rho_{u \mathfrak{U}}^{+}(\widetilde{\varrho}) \rho_{u \mathfrak{Q}}^{+}(\widetilde{\varrho})\right],\left[-\left(\rho_{\ell \mathfrak{U}}^{-}(\widetilde{\varrho}) \rho_{\ell \mathfrak{Q}}^{-}\right.\right.$ $\left.(\widetilde{\varrho})),-\left(\rho_{u \mathfrak{U}}^{-}(\widetilde{\varrho}) \rho_{u \mathfrak{Q}}^{-}(\widetilde{\varrho})\right)\right],\left(\rho_{\mathfrak{U}}^{+}(\widetilde{\varrho})+\rho_{\mathfrak{Q}}^{+}(\widetilde{\varrho})-\rho_{\mathfrak{U}}^{+} \quad(\widetilde{\varrho})\right.$ $\left.\left.\left.\rho_{\mathfrak{Q}}^{+}(\widetilde{\varrho}),-\left(\rho_{\mathfrak{U}}^{-}(\widetilde{\varrho}) \rho_{\mathfrak{Q}}^{-}(\widetilde{\varrho})\right)\right)\right\rangle: \widetilde{\varrho} \in \mathfrak{R}\right\}$

(iv) $\mathfrak{U} \otimes_{P} \mathfrak{Q}=\left\{\left\langle\widetilde{\varrho},\left[\rho_{\ell \mathfrak{U}}^{+}(\widetilde{\varrho}) \rho_{\ell \mathfrak{Q}}^{+}(\widetilde{\varrho}), \quad \rho_{u \mathfrak{U}}^{+}(\widetilde{\varrho}) \rho_{u \mathfrak{Q}}^{+}(\widetilde{\varrho})\right]\right.\right.$, $\left[-\left(-\rho_{\ell \mathfrak{U}}^{-}(\widetilde{\varrho})-\rho_{\ell \mathfrak{Q}}^{-}(\widetilde{\varrho})-\left(\rho_{\ell \mathfrak{U}}^{-}(\widetilde{\varrho}) \rho_{\ell \mathfrak{Q}}^{-}(\widetilde{\varrho})\right)\right), \quad-\left(-\rho_{u \mathfrak{U}}^{-}\right.\right.$ $\left.\left.(\widetilde{\varrho})-\rho_{u \mathfrak{Q}}^{-}(\widetilde{\varrho})-\left(\rho_{u \mathfrak{U}}^{-}(\widetilde{\varrho}) \rho_{u \mathfrak{Q}}^{-}(\widetilde{\varrho})\right)\right)\right],\left(\rho_{\mathfrak{U}}^{+}(\widetilde{\varrho}) \rho_{\mathfrak{Q}}^{+}(\widetilde{\varrho}),-\right.$ $\left.\left.\left.\left(-\rho_{\mathfrak{U}}^{-}(\widetilde{\varrho})-\rho_{\mathfrak{Q}}^{-}(\widetilde{\varrho})-\left(\rho_{\mathfrak{U}}^{-}(\widetilde{\varrho}) \rho_{\mathfrak{Q}}^{-}(\widetilde{\varrho})\right)\right)\right)\right\rangle: \widetilde{\varrho} \in \mathfrak{R}\right\}$

(v) $\mathfrak{U}^{\lambda}=\left\{\left\langle r,\left[\left(\rho_{\mathfrak{\ell} \mathfrak{U}}^{+}(\widetilde{\varrho})\right)^{\lambda},\left(\rho_{\mathfrak{u} \mathfrak{U}}^{+}(\widetilde{\varrho})\right)^{\lambda}\right], \quad[-(1-(1-\right.\right.$ $\left.\left.\left.\left(-\rho_{\mathfrak{\ell U}}^{-}(\widetilde{\varrho})\right)\right)^{\lambda}\right),-\left(1-\left(1-\left(-\rho_{\mathfrak{u} \mathfrak{U}}^{-}(\widetilde{\varrho})\right)\right)^{\lambda}\right)\right],\left(\left(\rho_{\mathfrak{U}}^{+}(\widetilde{\varrho})\right)^{\lambda}\right.$, $\left.\left.\left.-\left(1-\left(1-\left(-\rho_{\mathfrak{U}}^{-}(\widetilde{\varrho})\right)\right)^{\lambda}\right)\right)\right\rangle: \widetilde{\varrho} \in \mathfrak{R}\right\}$

(vi) $\lambda \mathfrak{U}=\left\{\left\langle\widetilde{\varrho},\left[1-\left(1-\rho_{\ell \mathfrak{U}}^{+}(\widetilde{\varrho})\right)^{\lambda}, \quad 1-\left(1-\rho_{u \mathfrak{U}}^{+}(\widetilde{\varrho})\right)^{\lambda}\right]\right.\right.$, $\left[-\left(-\rho_{\mathfrak{\ell} \mathfrak{U}}^{-}(\widetilde{\varrho})\right)^{\lambda}, \quad-\left(-\rho_{\mathfrak{u} \mathfrak{U}}^{-}(\widetilde{\varrho})\right)^{\lambda}\right],\left(1-\left(1-\rho_{\mathfrak{U}}^{+}(\widetilde{\varrho})\right)^{\lambda}\right.$, $\left.\left.\left.-\left(-\rho_{\mathfrak{U}}^{-}(\widetilde{\varrho})\right)^{\lambda}\right)\right\rangle: \widetilde{\varrho} \in \mathfrak{R}\right\}$

(vii) $\mathfrak{U} \subseteq_{P} \mathfrak{Q}$ if $\left[\rho_{\ell \mathfrak{U}}^{+}(\widetilde{\varrho}), \rho_{u \mathfrak{U}}^{+}(\widetilde{\varrho})\right] \leq\left[\rho_{\ell \mathfrak{Q}}^{+}(\widetilde{\varrho}), \rho_{u \mathfrak{Q}}^{+}(\widetilde{\varrho})\right]$ and $\left[\rho_{\ell \mathfrak{U}}^{-}(\widetilde{\varrho}), \rho_{u \mathfrak{U}}^{-}(\widetilde{\varrho})\right] \geq\left[\rho_{\ell \mathfrak{Q}}^{-}(\widetilde{\varrho}), \rho_{u \mathfrak{Q}}^{-}(\widetilde{\varrho})\right], \quad \rho_{\mathfrak{U}}^{+}(\widetilde{\varrho}) \leq$ $\rho_{\mathfrak{Q}}^{+}(\widetilde{\varrho})$ and $\rho_{\mathfrak{U}}^{-}(\widetilde{\varrho}) \geq \rho_{\mathfrak{Q}}^{-}(\widetilde{\varrho}), \forall \widetilde{\varrho} \in \mathfrak{R}$

Definition 3 (see [37]). Let $\mathcal{U}=\left\{\left\langle\widetilde{\varrho},\left[\rho_{\ell \mathfrak{U}}^{+}(\widetilde{\varrho}), \rho_{\mathfrak{u} \mathfrak{U}}^{+}(\widetilde{\varrho})\right]\right.\right.$, $\left.\left.\left[\rho_{\ell \mathfrak{U}}^{-}(\widetilde{\varrho}), \rho_{u \mathfrak{U}}^{-}(\widetilde{\varrho})\right],\left(\rho_{\mathfrak{U}}^{+}(\widetilde{\varrho}), \rho_{\mathfrak{U}}^{-}(\widetilde{\varrho})\right)\right\rangle: \widetilde{\varrho} \in \mathfrak{R}\right\}$ and $\mathfrak{Q}=\{\langle\widetilde{\varrho}$, $\left.\left[\rho_{\ell \mathfrak{Q}}^{+}(\widetilde{\varrho}), \rho_{u \mathfrak{Q}}^{+}(\widetilde{\varrho})\right], \quad\left[\rho_{\ell \mathfrak{Q}}^{-}(\widetilde{\varrho}), \quad \rho_{u \mathfrak{Q}}^{-}(\widetilde{\varrho})\right], \quad\left(\rho_{\mathfrak{Q}}^{+}(\widetilde{\varrho}), \rho_{\mathfrak{Q}}^{-}(\widetilde{\varrho})\right)\right\rangle:$ $\widetilde{\varrho} \in \mathfrak{R}\}$ be two CBFSs on $\mathfrak{R}$ and $\lambda>0$. Then, we have the following:

(i) $\mathfrak{R} \cup_{R} \mathfrak{Q}=\left\{\left\langle\widetilde{\varrho},\left[\max \left\{\rho_{\ell \mathfrak{U}}^{+}(\widetilde{\varrho}), \quad \rho_{\ell \mathfrak{Q}}^{+}(\widetilde{\varrho})\right\}, \max \left\{\rho_{u \mathfrak{U}}^{+}\right.\right.\right.\right.$ $\left.\left.(\widetilde{\varrho}), \rho_{u \mathfrak{Q}}^{+}(\widetilde{\varrho})\right\}\right],\left[\min \left\{\rho_{\ell \mathfrak{U}}^{-}(\widetilde{\varrho}), \rho_{\ell \mathfrak{Q}}^{-}(\widetilde{\varrho})\right\}, \min \left\{\rho_{u \mathfrak{U}}^{-}(\widetilde{\varrho})\right.\right.$, $\left.\left.\rho_{u \mathfrak{Q}}^{-}(\widetilde{\varrho})\right\}\right], \quad\left(\min \left\{\rho_{\mathfrak{U}}^{+}(\widetilde{\varrho}), \rho_{\mathfrak{Q}}^{+}(\widetilde{\varrho})\right\}, \max \left\{\rho_{\mathfrak{U}}^{-}(\widetilde{\varrho}), \rho_{\mathfrak{Q}}^{-}\right.\right.$ $(\widetilde{\varrho})\})\rangle: \widetilde{\varrho} \in \mathfrak{R}\}$

(ii) $\mathfrak{U} \cap_{R} \mathfrak{Q}=\left\{\left\langle\widetilde{\varrho}, \quad\left[\min \left\{\rho_{\ell \mathfrak{U}}^{+}(\widetilde{\varrho}), \rho_{\ell \mathfrak{Q}}^{+}(\widetilde{\varrho})\right\}, \quad \min \left\{\rho_{u \mathfrak{U}}^{+}\right.\right.\right.\right.$ $\left.\left.(\widetilde{\varrho}), \rho_{u \mathfrak{Q}}^{+}(\widetilde{\varrho})\right\}\right],\left[\max \left\{\rho_{\ell \mathfrak{U}}^{-}(\widetilde{\varrho}), \rho_{\ell \mathfrak{Q}}^{-}(\widetilde{\varrho})\right\}, \max \left\{\rho_{u \mathfrak{U}}^{-}(\widetilde{\varrho})\right.\right.$, $\left.\left.\rho_{u \mathfrak{Q}}^{-}(\widetilde{\varrho})\right\}\right],\left(\max \left\{\rho_{\mathfrak{U}}^{+}(\widetilde{\varrho}), \quad \rho_{\mathfrak{Q}}^{+}(\widetilde{\varrho})\right\}, \min \left\{\rho_{\mathfrak{U}}^{-}(\widetilde{\varrho}), \rho_{\mathfrak{Q}}^{-}\right.\right.$ $(\widetilde{\varrho})\})\rangle: \widetilde{\varrho} \in \mathfrak{R}\}$

(iii) $\mathfrak{U} \oplus_{R} \mathfrak{Q}=\left\{\left\langle\widetilde{\varrho},\left[\rho_{\ell \mathfrak{U}}^{+}(\widetilde{\varrho})+\rho_{\ell \mathfrak{Q}}^{+}(\widetilde{\varrho})-\rho_{\ell \mathfrak{U}}^{+}(\widetilde{\varrho}) \rho_{\ell \mathfrak{Q}}^{+} \quad(\widetilde{\varrho})\right.\right.\right.$,

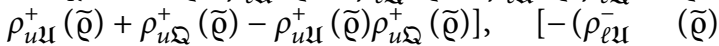
$\left.\left.\rho_{\ell \mathfrak{Q}}^{-}(\widetilde{\varrho})\right),-\left(\rho_{u \mathfrak{U}}^{-}(\widetilde{\varrho}) \rho_{u \mathfrak{Q}}^{-}(\widetilde{\varrho})\right)\right],\left(\rho_{\mathfrak{U}}^{+} \quad(\widetilde{\varrho}) \rho_{\mathfrak{Q}}^{+}(\widetilde{\varrho}),-\right.$ $\left.\left.\left.\left(-\rho_{\mathfrak{U}}^{-}(\widetilde{\varrho})-\rho_{\mathfrak{Q}}^{-}(\widetilde{\varrho})-\left(\rho_{\mathfrak{U}}^{-}(\widetilde{\varrho}) \rho_{\mathfrak{Q}}^{-}(\widetilde{\varrho})\right)\right)\right)\right\rangle: \widetilde{\varrho} \in \mathfrak{R}\right\}$

(iv) $\mathfrak{U} \otimes_{R} \mathfrak{Q}=\left\{\left\langle\widetilde{\varrho},\left[\rho_{\ell \mathfrak{U}}^{+}(\widetilde{\varrho}) \rho_{\ell \mathfrak{Q}}^{+}(\widetilde{\varrho}), \rho_{u \mathfrak{U}}^{+}(\widetilde{\varrho}) \rho_{u \mathfrak{Q}}^{+}(\widetilde{\varrho})\right],[-\right.\right.$ $\left(-\rho_{\ell \mathfrak{U}}^{-}(\widetilde{\mathrm{Q}})-\rho_{\ell \mathfrak{Q}}^{-}(\widetilde{\mathrm{Q}})-\left(\rho_{\ell \mathfrak{U}}^{-}(\widetilde{\mathrm{Q}}) \rho_{\ell \mathfrak{Q}}^{-}(\widetilde{\mathrm{Q}})\right)\right),-\left(-\rho_{u \mathfrak{U}}^{-}(\widetilde{\mathrm{Q}})-\right.$ $\left.\left.\rho_{\mathcal{u} \mathfrak{Q}}^{-}(\widetilde{\varrho})-\left(\rho_{u \mathfrak{U}}^{-}(\widetilde{\varrho}) \rho_{u \mathfrak{Q}}^{-}(\widetilde{\varrho})\right)\right)\right], \quad\left(\rho_{\mathfrak{U}}^{+}(\widetilde{\varrho})+\rho_{\mathfrak{Q}}^{+}(\widetilde{\varrho})-\rho_{\mathfrak{U}}^{+}\right.$ $\left.\left.\left.(\widetilde{\varrho}) \rho_{\mathfrak{Q}}^{+}(\widetilde{\varrho}),-\left(\rho_{\mathfrak{U}}^{-}(\widetilde{\varrho}) \rho_{\mathfrak{Q}}^{-}(\widetilde{\varrho})\right)\right)\right\rangle: \widetilde{\varrho} \in \mathfrak{R}\right\}$

(v) $\mathfrak{U}^{\lambda}=\left\{\left\langle r,\left[\left(\rho_{\ell \mathfrak{U}}^{+}(\widetilde{\varrho})\right)^{\lambda},\left(\rho_{\mathfrak{u} \mathfrak{U}}^{+}(\widetilde{\varrho})\right)^{\lambda}\right],\left[-\left(1-\left(1-\left(-\rho_{\ell \mathfrak{U}}^{-}\right.\right.\right.\right.\right.\right.$ $\left.\left.(\widetilde{\varrho})))^{\lambda}\right),-\left(1-\left(1-\left(-\rho_{\mathfrak{u} \mathfrak{U}}^{-}(\widetilde{\varrho})\right)\right)^{\lambda}\right)\right], \quad\left(1-\left(1-\rho_{\mathfrak{U}}^{+}\right.\right.$ $\left.\left.\left.(\widetilde{\varrho}))^{\lambda},-\left(-\rho_{\mathfrak{U}}^{-}(\widetilde{\varrho})\right)^{\lambda}\right)\right\rangle: \widetilde{\varrho} \in \mathfrak{R}\right\}$

(vi) $\lambda \mathfrak{U}=\left\{\left\langle\widetilde{\varrho},\left[1-\left(1-\rho_{\ell \mathfrak{U}}^{+}(\widetilde{\varrho})\right)^{\lambda}, 1-\left(1-\rho_{u \mathfrak{U}}^{+}(\widetilde{\varrho})\right)^{\lambda}\right], \quad[-\right.\right.$ $\left.\left(-\rho_{\mathfrak{\ell} \mathfrak{U}}^{-}(\widetilde{\varrho})\right)^{\lambda},-\left(-\rho_{\mathfrak{u} \mathfrak{U}}^{-}(\widetilde{\varrho})\right)^{\lambda}\right],\left(\left(\rho_{\mathfrak{U}}^{+}(\widetilde{\varrho})\right)^{\lambda},-(1-(1-\right.$ $\left.\left.\left.\left.\left(-\rho_{\mathfrak{U}}^{-}(\widetilde{\varrho})\right)^{\lambda}\right)\right)\right\rangle: \widetilde{\varrho} \in \mathfrak{R}\right\}$

(vii) $\mathfrak{U} \subseteq{ }_{R} \mathfrak{Q}$ if $\left[\rho_{\ell \mathfrak{U}}^{+}(\widetilde{\varrho}), \rho_{u \mathfrak{U}}^{+}(\widetilde{\varrho})\right] \leq\left[\rho_{\ell \mathfrak{Q}}^{+}(\widetilde{\varrho}), \rho_{u \mathfrak{Q}}^{+}(\widetilde{\varrho})\right]$ and $\left[\rho_{\ell \mathfrak{U}}^{-}(\widetilde{\mathrm{Q}}), \rho_{u \mathfrak{U}}^{-}(\widetilde{\mathrm{Q}})\right] \geq\left[\rho_{\ell \mathfrak{Q}}^{-}(\widetilde{\mathrm{Q}}), \rho_{\mathcal{u Q}}^{-}(\widetilde{\mathrm{Q}})\right], \quad \rho_{\mathfrak{U}}^{+}(\widetilde{\mathrm{Q}}) \geq \rho_{\mathfrak{Q}}^{+}$ $(\widetilde{\varrho})$ and $\rho_{\mathfrak{U}}^{-}(\widetilde{\varrho}) \leq \rho_{\mathfrak{Q}}^{-}(\widetilde{\varrho}), \forall \widetilde{\varrho} \in \mathfrak{R}$

\section{Cosine Similarity Measures for CBFSs}

In this section, we define three cosine SMs for CBFSs. We examine their properties and give examples for better understanding. Later on, the weighted versions of these similarity measures will also be presented.

Definition 4. Let $\mathfrak{R}=\left\{\widetilde{\varrho}_{1}, \widetilde{\varrho}_{2}, \ldots, \widetilde{\varrho}_{n}\right\}$ be a finite universe of discourse.

Let $\quad \mathcal{U}=\left\{\left\langle\widetilde{\varrho}_{i},\left[\rho_{\ell \mathfrak{U}}^{+}\left(\widetilde{\varrho}_{i}\right), \rho_{u \mathfrak{U}}^{+} \quad\left(\widetilde{\varrho}_{i}\right)\right],\left[\rho_{\ell \mathfrak{U}}^{-}\left(\widetilde{\varrho}_{i}\right), \rho_{u \mathfrak{U}}^{-}\left(\widetilde{\varrho}_{i}\right)\right]\right.\right.$, $\left.\left.\left(\rho_{\mathfrak{U}}^{+}\left(\widetilde{\varrho}_{i}\right), \rho_{\mathfrak{U}}^{-}\left(\widetilde{\varrho}_{i}\right)\right)\right\rangle: \widetilde{\varrho}_{i} \in \mathfrak{R}\right\}$ and $\mathfrak{H}=\left\{\left\langle\widetilde{\varrho}_{i},\left[\rho_{\ell \mathfrak{T}}^{+}\left(\widetilde{\varrho}_{i}\right), \rho_{u \mathfrak{T}}^{+}\right.\right.\right.$ $\left.\left.\left.\left(\widetilde{\varrho}_{i}\right)\right],\left[\rho_{\ell \mathfrak{H}}^{-}\left(\widetilde{\varrho}_{i}\right), \rho_{u \mathfrak{H}}^{-}\left(\widetilde{\varrho}_{i}\right)\right],\left(\rho_{\mathfrak{H}}^{+}\left(\widetilde{\varrho}_{i}\right), \rho_{\mathfrak{H}}^{-}\left(\widetilde{\varrho}_{i}\right)\right)\right\rangle: \widetilde{\varrho}_{i} \in \mathfrak{R}\right\}$ be two CBFSs on $\Re$; then, cosine SM based on the cosine of the angle between two vectors is given by

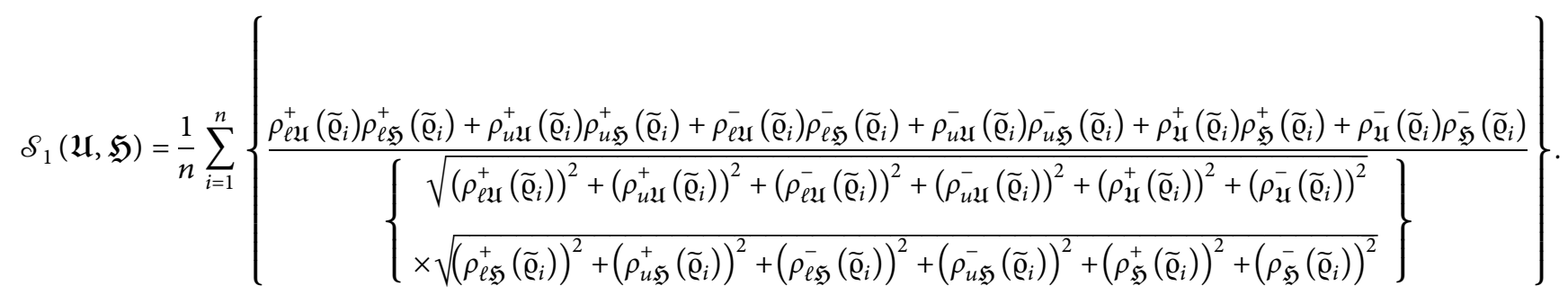


Theorem 1. For two CBFSs $\mathfrak{U}$ and $\mathfrak{H}$, the cosine SM proposed in equation (3) possesses the following properties:

$$
\begin{aligned}
& \text { (i) } 0 \leq \mathcal{S}_{1}(\mathfrak{U}, \mathfrak{H}) \leq 1 \\
& \text { (ii) } \mathcal{S}_{1}(\mathfrak{U}, \mathfrak{H})=\mathcal{S}_{1}(\mathfrak{H}, \mathfrak{U}) \\
& \text { (iii) } \mathcal{S}_{1}(\mathfrak{U}, \mathfrak{H})=1 \text { if } \mathfrak{U}=\mathfrak{H}
\end{aligned}
$$

Proof. (i) It is obvious that $\mathcal{S}_{1}(\mathfrak{U}, \mathfrak{H}) \geq 0$. We only have to show that $\mathcal{S}_{1}(\mathfrak{U}, \mathfrak{H}) \leq 1$. The Cauchy-Schwarz inequality further implies that

$$
\begin{aligned}
\left(a_{1} b_{1}+a_{2} b_{2}+\cdots+a_{n} b_{n}\right) \leq & \sqrt{\left(a_{1}^{2}+a_{2}^{2}+\cdots+a_{n}^{2}\right)} \\
& \times \sqrt{\left(b_{1}^{2}+b_{2}^{2}+\cdots+b_{n}^{2}\right)} .
\end{aligned}
$$

Utilizing the above inequality, we have

$$
\rho_{\ell \mathfrak{U}}^{+}\left(\widetilde{\varrho}_{i}\right) \rho_{\ell \mathfrak{T}}^{+}\left(\widetilde{\varrho}_{i}\right)+\rho_{u \mathfrak{U}}^{+}\left(\widetilde{\varrho}_{i}\right) \rho_{u \mathfrak{Y}}^{+}\left(\widetilde{\varrho}_{i}\right)+\rho_{l \mathfrak{U}}^{-}\left(\widetilde{\varrho}_{i}\right) \rho_{\ell \mathfrak{Y}}^{-}\left(\widetilde{\varrho}_{i}\right)+\rho_{u \mathfrak{U}}^{-}\left(\widetilde{\varrho}_{i}\right) \rho_{u \mathfrak{Y}}^{-}\left(\widetilde{\varrho}_{i}\right)+\rho_{\mathfrak{U}}^{+}\left(\widetilde{\varrho}_{i}\right) \rho_{\mathfrak{H}}^{+}\left(\widetilde{\varrho}_{i}\right)+\rho_{\mathfrak{U}}^{-}\left(\widetilde{\varrho}_{i}\right) \rho_{\mathfrak{Y}}^{-}\left(\widetilde{\varrho}_{i}\right)
$$

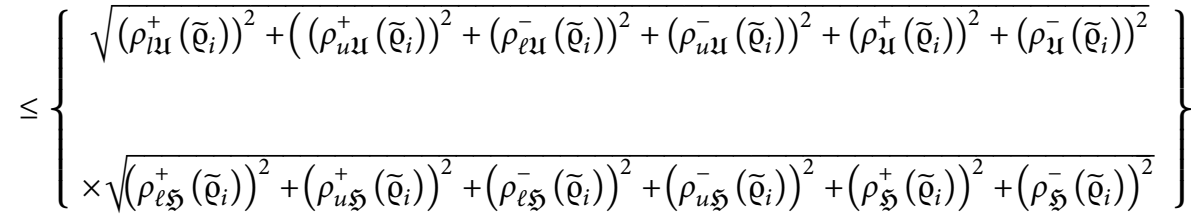

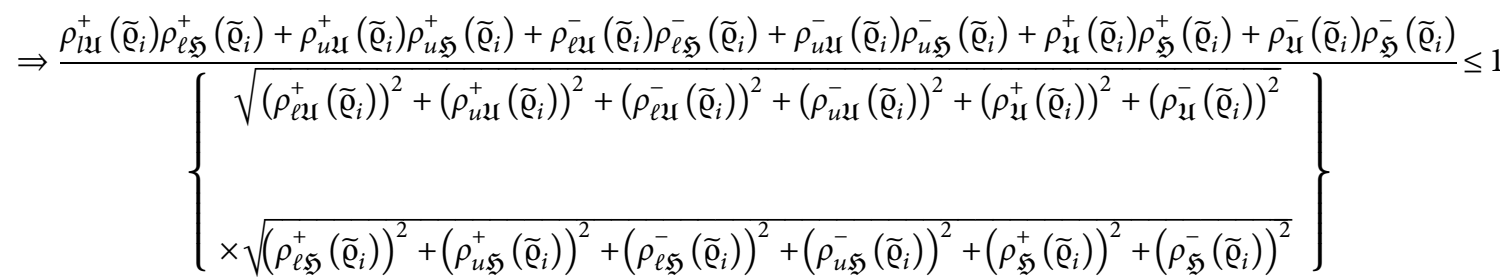

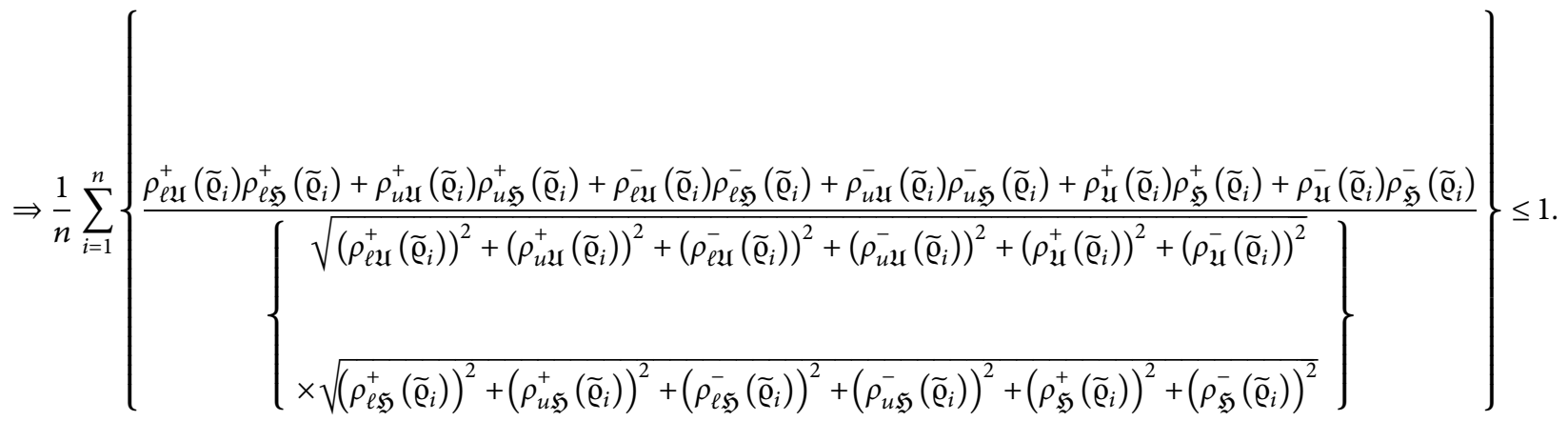

Hence, $0 \leq \mathcal{S}_{1}(\mathfrak{U}, \mathfrak{H}) \leq 1$.

(ii) It is obvious.

(iii) If $\mathfrak{U}=\mathfrak{H}$, then $\left[\rho_{\ell \mathfrak{U}}^{+}\left(\widetilde{\varrho}_{i}\right), \rho_{u \mathfrak{U}}^{+}\left(\widetilde{\varrho}_{i}\right)\right]=\left[\rho_{\ell \mathfrak{H}}^{+}\left(\widetilde{\varrho}_{i}\right)\right.$, $\left.\rho_{u \mathfrak{H}}^{+}\left(\widetilde{\varrho}_{i}\right)\right], \quad\left[\rho_{\ell \mathfrak{U}}^{-}\left(\widetilde{\varrho}_{i}\right), \rho_{u \mathfrak{U}}^{-}\left(\widetilde{\varrho}_{i}\right)\right]=\left[\rho_{\ell \mathfrak{H}}^{-}\left(\widetilde{\varrho}_{i}\right), \rho_{u \mathfrak{H}}^{-}\left(\widetilde{\varrho}_{i}\right)\right]$, $\rho_{\ell \mathfrak{U}}^{+}\left(\widetilde{\mathrm{Q}}_{i}\right)=\rho_{\ell \mathfrak{S}}^{+}\left(\widetilde{\mathrm{Q}}_{i}\right)$, and $\rho_{\ell \mathfrak{U}}^{-}\left(\widetilde{\mathrm{Q}}_{i}\right)=\rho_{\ell \mathfrak{S}}^{-}\left(\widetilde{\mathrm{Q}}_{i}\right)$, for all $\widetilde{\varrho}_{i} \in \mathfrak{R}$. Thus, $\mathcal{S}_{1}(\mathfrak{U}, \mathfrak{H})=1$.
Example 1 . Let $\mathfrak{U}=\left\{\left\langle\widetilde{\varrho}_{1},[0.11,0.26],[-0.38,-0.23]\right.\right.$, $(0.12,-0.31)\rangle,\left\langle\widetilde{\varrho}_{2},[0.22,0.37], \quad[-0.44,-0.29], \quad(0.23\right.$, $-0.42)\rangle\}$ and $\mathfrak{H}=\left\{\left\langle\widetilde{\varrho}_{1},[0.61,0.76],[-0.59,-0.44], \quad(0.73\right.\right.$, $\left.-0.41)\rangle,\left\langle\widetilde{\varrho}_{2},[0.59,0.74],[-0.29,-0.14],(0.72,-0.11)\right\rangle\right\}$ be two CBFSs on $\mathfrak{R}=\left\{\widetilde{\varrho}_{1}, \widetilde{\varrho}_{2}\right\}$. Then, by utilizing equation (3), we calculate the cosine similarity measure between $\mathcal{U}$ and $\mathfrak{H}$ as 


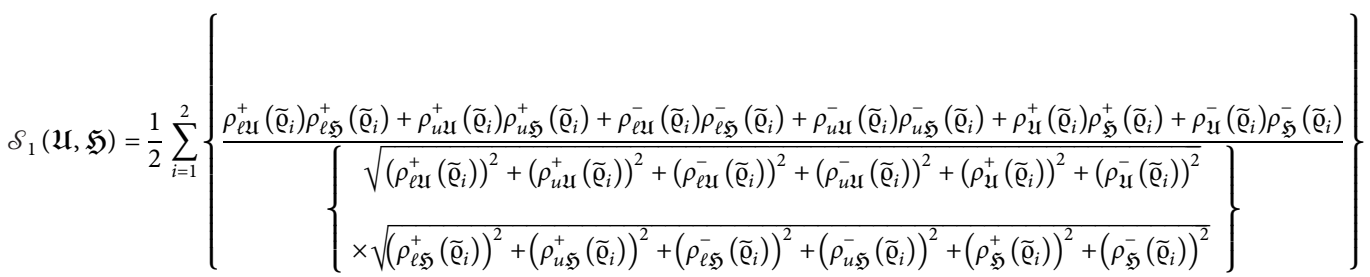

$$
\begin{aligned}
& =\frac{1}{2}\left\{\left(\frac{(0.11 \times 0.61)+(0.26 \times 0.76)+(-0.38 \times-0.59)+(-0.23 \times-0.44)+(0.12 \times 0.73)+(-0.31 \times-0.41)}{\sqrt{(0.11)^{2}+(0.26)^{2}+(-0.38)^{2}+(-0.23)^{2}+(0.12)^{2}+(-0.31)^{2}} \times \sqrt{(0.61)^{2}+(0.76)^{2}+(-0.59)^{2}+(-0.44)^{2}+(0.73)^{2}+(-0.41)^{2}}}\right)\right. \\
& \left.+\left(\frac{(0.22 \times 0.59)+(0.37 \times 0.74)+(-0.44 \times-0.29)+(-0.29 \times-0.14)+(0.23 \times 0.72)+(-0.42 \times-0.11)}{\sqrt{(0.22)^{2}+(0.37)^{2}+(-0.44)^{2}+(-0.29)^{2}+(0.23)^{2}+(-0.42)^{2}} \times \sqrt{(0.59)^{2}+(0.74)^{2}+(-0.29)^{2}+(-0.14)^{2}+(0.72)^{2}+(-0.11)^{2}}}\right)\right\} \\
& =0.8173 \text {. }
\end{aligned}
$$

Definition 5. Let $\Re=\left\{\widetilde{\varrho}_{1}, \widetilde{\varrho}_{2}, \ldots, \widetilde{\varrho}_{n}\right\}$ be a finite universe of discourse, and let $\mathfrak{U}=\left\{\left\langle\widetilde{\varrho}_{i},\left[\rho_{\ell \mathfrak{U}}^{+}\left(\widetilde{\varrho}_{i}\right), \rho_{\mathfrak{u} \mathfrak{U}}^{+}\left(\widetilde{\varrho}_{i}\right)\right],\left[\rho_{\ell \mathfrak{U}}^{-}\left(\widetilde{\varrho}_{i}\right)\right.\right.\right.$, $\left.\left.\left.\rho_{u \mathfrak{U}}^{-}\left(\widetilde{\varrho}_{i}\right)\right],\left(\rho_{\mathfrak{U}}^{+}\left(\widetilde{\varrho}_{i}\right), \rho_{\mathfrak{U}}^{-}\left(\widetilde{\varrho}_{i}\right)\right)\right\rangle: \widetilde{\varrho}_{i} \in \mathfrak{R}\right\}$ and $\mathfrak{S}=\left\{\left\langle\widetilde{\varrho}_{i},\left[\rho_{\ell \mathfrak{H}}^{+}\left(\widetilde{\varrho}_{i}\right)\right.\right.\right.$, $\left.\left.\left.\rho_{u \mathfrak{T}}^{+}\left(\widetilde{\varrho}_{i}\right)\right],\left[\rho_{\ell \mathfrak{H}}^{-}\left(\widetilde{\varrho}_{i}\right), \rho_{u \mathfrak{H}}^{-}\left(\widetilde{\varrho}_{i}\right)\right],\left(\rho_{\mathfrak{S}}^{+}\left(\widetilde{\varrho}_{i}\right), \rho_{\mathfrak{H}}^{-}\left(\widetilde{\varrho}_{i}\right)\right)\right\rangle: \widetilde{\varrho}_{i} \in \mathfrak{R}\right\}$ be two CBFSs on $\mathfrak{R}$; then, cosine SM based on distance is given by

$$
\mathcal{S}_{2}(\mathfrak{U}, \mathfrak{H})=\frac{1}{n} \sum_{i=1}^{n} \cos \left\{\begin{array}{l}
\left(\left|\rho_{\ell \mathfrak{U}}^{+}\left(\widetilde{\varrho}_{i}\right)-\rho_{\ell \mathfrak{W}}^{+}\left(\widetilde{\varrho}_{i}\right)\right|+\left|\rho_{u \mathfrak{U}}^{+}\left(\widetilde{\varrho}_{i}\right)-\rho_{u \mathfrak{W}}^{+}\left(\widetilde{\varrho}_{i}\right)\right|+\left|\rho_{\ell \mathfrak{U}}^{-}\left(\widetilde{\varrho}_{i}\right)-\rho_{\ell \mathfrak{W}}^{-}\left(\widetilde{\varrho}_{i}\right)\right|\right. \\
\left.+\left|\rho_{u \mathfrak{U}}^{-}\left(\widetilde{\varrho}_{i}\right)-\rho_{u \mathfrak{H}}^{-}\left(\widetilde{\varrho}_{i}\right)\right|+\left|\rho_{\mathfrak{U}}^{+}\left(\widetilde{\varrho}_{i}\right)-\rho_{\mathfrak{H}}^{+}\left(\widetilde{\varrho}_{i}\right)\right|+\left|\rho_{\mathfrak{U}}^{-}\left(\widetilde{\varrho}_{i}\right)-\rho_{\mathfrak{H}}^{-}\left(\widetilde{\varrho}_{i}\right)\right|\right) \frac{\pi}{12}
\end{array}\right\} .
$$

Theorem 2. For two CBFSs $\mathfrak{U}$ and $\mathfrak{H}$, the cosine similarity measure proposed in equation (7) possesses the following properties:

(i) $0 \leq \mathcal{S}_{2}(\mathfrak{U}, \mathfrak{Y}) \leq 1$

(ii) $\mathcal{S}_{2}(\mathfrak{U}, \mathfrak{H})=\mathcal{S}_{2}(\mathfrak{H}, \mathfrak{U})$

(iii) $\mathcal{S}_{2}(\mathfrak{U}, \mathfrak{H})=1$ iff $\mathfrak{U}=\mathfrak{H}$
Proof

(i) We know that $0 \leq\left|\rho_{\ell \mathfrak{U}}^{+}\left(\widetilde{\varrho}_{i}\right)-\rho_{\ell \mathfrak{T}}^{+}\left(\widetilde{\varrho}_{i}\right)\right| \leq 1, \quad 0 \leq$ $\left|\rho_{u \mathfrak{U}}^{+}\left(\widetilde{\varrho}_{i}\right)-\rho_{u \mathfrak{H}}^{+}\left(\widetilde{\varrho}_{i}\right)\right| \leq 1, \quad 0 \leq\left|\rho_{\ell \mathfrak{U}}^{-}\left(\widetilde{\varrho}_{i}\right)-\rho_{\ell \mathfrak{H}}^{-}\left(\widetilde{\varrho}_{i}\right)\right| \leq 1$, $0 \leq\left|\rho_{u \mathfrak{U}}^{-}\left(\widetilde{\varrho}_{i}\right)-\rho_{u \mathfrak{H}}^{-}\left(\widetilde{\varrho}_{i}\right)\right| \leq 1,0 \leq\left|\rho_{\mathfrak{U}}^{+}\left(\widetilde{\varrho}_{i}\right)-\rho_{\mathfrak{S}}^{+}\left(\widetilde{\varrho}_{i}\right)\right| \leq 1$, and $0 \leq\left|\rho_{\mathfrak{U}}^{-}\left(\widetilde{\varrho}_{i}\right)-\rho_{\mathfrak{S}}^{-}\left(\widetilde{\varrho}_{i}\right)\right| \leq 1$, for all $\widetilde{\varrho}_{i} \in \Re$.

By combining all these inequalities, we get

$$
\begin{aligned}
& 0 \leq\left|\rho_{\ell \mathfrak{U}}^{+}\left(\widetilde{\varrho}_{i}\right)-\rho_{\ell \mathfrak{Y}}^{+}\left(\widetilde{\varrho}_{i}\right)\right|+\left|\rho_{u \mathfrak{U}}^{+}\left(\widetilde{\varrho}_{i}\right)-\rho_{u \mathfrak{S}}^{+}\left(\widetilde{\varrho}_{i}\right)\right|+\left|\rho_{\ell \mathfrak{U}}^{-}\left(\widetilde{\varrho}_{i}\right)-\rho_{\ell \mathfrak{W}}^{-}\left(\widetilde{\varrho}_{i}\right)\right|+\left|\rho_{u \mathfrak{U}}^{-}\left(\widetilde{\varrho}_{i}\right)-\rho_{u \mathfrak{S}}^{-}\left(\widetilde{\varrho}_{i}\right)\right| \\
& +\left|\rho_{\mathfrak{U}}^{+}\left(\widetilde{\varrho}_{i}\right)-\rho_{\mathfrak{S}}^{+}\left(\widetilde{\varrho}_{i}\right)\right|+\left|\rho_{\mathfrak{U}}^{-}\left(\widetilde{\varrho}_{i}\right)-\rho_{\mathfrak{J}}^{-}\left(\widetilde{\varrho}_{i}\right)\right| \leq 6 \\
& \Rightarrow 0 \leq \frac{1}{6}\left(\left|\rho_{\ell \mathfrak{U}}^{+}\left(\widetilde{\varrho}_{i}\right)-\rho_{\ell \mathfrak{W}}^{+}\left(\widetilde{\varrho}_{i}\right)\right|+\left|\rho_{u \mathfrak{U}}^{+}\left(\widetilde{\varrho}_{i}\right)-\rho_{u \mathfrak{W}}^{+}\left(\widetilde{\varrho}_{i}\right)\right|+\left|\rho_{\ell \mathfrak{U}}^{-}\left(\widetilde{\varrho}_{i}\right)-\rho_{\ell \mathfrak{S}}^{-}\left(\widetilde{\varrho}_{i}\right)\right|+\left|\rho_{u \mathfrak{U}}^{-}\left(\widetilde{\varrho}_{i}\right)-\rho_{u \mathfrak{N}}^{-}\left(\widetilde{\varrho}_{i}\right)\right|\right. \\
& \left.+\left|\rho_{\mathfrak{U}}^{+}\left(\widetilde{\varrho}_{i}\right)-\rho_{\mathfrak{H}}^{+}\left(\widetilde{\varrho}_{i}\right)\right|+\left|\rho_{\mathfrak{U}}^{-}\left(\widetilde{\varrho}_{i}\right)-\rho_{\mathfrak{H}}^{-}\left(\widetilde{\varrho}_{i}\right)\right|\right) \leq 1 \\
& \Rightarrow 0 \leq \cos \left\{\frac { \pi } { 2 } \left(\frac { 1 } { 6 } \left(\left|\rho_{\ell \mathfrak{U}}^{+}\left(\widetilde{\varrho}_{i}\right)-\rho_{\ell \mathfrak{S}}^{+}\left(\widetilde{\varrho}_{i}\right)\right|+\left|\rho_{u \mathfrak{U}}^{+}\left(\widetilde{\varrho}_{i}\right)-\rho_{u \mathfrak{S}}^{+}\left(\widetilde{\varrho}_{i}\right)\right|+\left|\rho_{\ell \mathfrak{U}}^{-}\left(\widetilde{\varrho}_{i}\right)-\rho_{\ell \mathfrak{S}}^{-}\left(\widetilde{\varrho}_{i}\right)\right|+\left|\rho_{u \mathfrak{U}}^{-}\left(\widetilde{\varrho}_{i}\right)-\rho_{u \mathfrak{S}}^{-}\left(\widetilde{\varrho}_{i}\right)\right|\right.\right.\right. \\
& \left.\left.\left.+\left|\rho_{\mathfrak{U}}^{+}\left(\widetilde{\varrho}_{i}\right)-\rho_{\mathfrak{S}}^{+}\left(\widetilde{\varrho}_{i}\right)\right|+\left|\rho_{\mathfrak{U}}^{-}\left(\widetilde{\varrho}_{i}\right)-\rho_{\mathfrak{S}}^{-}\left(\widetilde{\varrho}_{i}\right)\right|\right)\right)\right\} \leq 1 \\
& \begin{array}{l}
\Rightarrow 0 \leq \frac{1}{n} \sum_{i=1}^{n} \cos \left\{\left(\left|\rho_{\ell \mathfrak{U}}^{+}\left(\widetilde{\varrho}_{i}\right)-\rho_{\ell \mathfrak{S}}^{+}\left(\widetilde{\varrho}_{i}\right)\right|+\left|\rho_{u \mathfrak{U}}^{+}\left(\widetilde{\varrho}_{i}\right)-\rho_{u \mathfrak{Y}}^{+}\left(\widetilde{\varrho}_{i}\right)\right|+\left|\rho_{\ell \mathfrak{U}}^{-}\left(\widetilde{\varrho}_{i}\right)-\rho_{\ell \mathfrak{W}}^{-}\left(\widetilde{\varrho}_{i}\right)\right|+\left|\rho_{u \mathfrak{U}}^{-}\left(\widetilde{\varrho}_{i}\right)-\rho_{u \mathfrak{W}}^{-}\left(\widetilde{\varrho}_{i}\right)\right| \leq 1 .\right.\right. \\
\left.\left.\left.+\widetilde{\varrho}_{i}\right)-\rho_{\mathfrak{S}}^{+}\left(\widetilde{\varrho}_{i}\right)|+| \rho_{\mathfrak{U}}^{-}\left(\widetilde{\varrho}_{i}\right)-\rho_{\mathfrak{S}}^{-}\left(\widetilde{\varrho}_{i}\right) \mid\right) \frac{\pi}{12}\right\}
\end{array}
\end{aligned}
$$


Hence, $0 \leq \mathcal{S}_{2}(\mathfrak{U}, \mathfrak{H}) \leq 1$.

(ii) It is obvious.

(iii) If $\mathfrak{U}=\mathfrak{H}$, then $\left[\rho_{l \mathfrak{U}}^{+}\left(\widetilde{\mathrm{Q}}_{i}\right), \rho_{u \mathfrak{L}}^{+}\left(\widetilde{\mathrm{Q}}_{i}\right)\right]=\left[\rho_{\ell \mathfrak{S}}^{+}\left(\widetilde{\mathrm{Q}}_{i}\right)\right.$, $\left.\rho_{u \mathfrak{S}}^{+}\left(\widetilde{\varrho}_{i}\right)\right], \quad\left[\rho_{\ell \mathfrak{U}}^{-}\left(\widetilde{\varrho}_{i}\right), \rho_{u \mathfrak{U}}^{-}\left(\widetilde{\varrho}_{i}\right)\right]=\left[\rho_{\ell \mathfrak{H}}^{-}\left(\widetilde{\varrho}_{i}\right), \rho_{u \mathfrak{H}}^{-}\left(\widetilde{\varrho}_{i}\right)\right]$, $\rho_{\mathfrak{U}}^{+}\left(\widetilde{\mathrm{Q}}_{i}\right)=\rho_{\mathfrak{S}}^{+}\left(\widetilde{\varrho}_{i}\right)$, and $\rho_{\mathfrak{U}}^{-}\left(\widetilde{\varrho}_{i}\right)=\rho_{\mathfrak{S}}^{-}\left(\widetilde{\mathfrak{Q}}_{i}\right)$, for all $\widetilde{\mathrm{Q}}_{i} \in \mathfrak{R}$. Therefore,

$$
\begin{aligned}
\left|\rho_{\ell \mathfrak{U}}^{+}\left(\widetilde{\varrho}_{i}\right)-\rho_{\ell \mathfrak{H}}^{+}\left(\widetilde{\varrho}_{i}\right)\right| & =0, \\
\left|\rho_{u \mathfrak{U}}^{+}\left(\widetilde{\varrho}_{i}\right)-\rho_{u \mathfrak{H}}^{+}\left(\widetilde{\varrho}_{i}\right)\right| & =0, \\
\left|\rho_{\ell \mathfrak{U}}^{-}\left(\widetilde{\varrho}_{i}\right)-\rho_{\ell \mathfrak{S}}^{-}\left(\widetilde{\varrho}_{i}\right)\right| & =0, \\
\left|\rho_{u \mathfrak{U}}^{-}\left(\widetilde{\varrho}_{i}\right)-\rho_{u \mathfrak{Y}}^{-}\left(\widetilde{\varrho}_{i}\right)\right| & =0, \\
\left|\rho_{\mathfrak{U}}^{+}\left(\widetilde{\varrho}_{i}\right)-\rho_{\mathfrak{H}}^{+}\left(\widetilde{\varrho}_{i}\right)\right| & =0, \\
\left|\rho_{\mathfrak{U}}^{+}\left(\widetilde{\varrho}_{i}\right)-\rho_{\mathfrak{H}}^{+}\left(\widetilde{\varrho}_{i}\right)\right| & =0, \quad \text { for all } \widetilde{\varrho}_{i} \in \mathfrak{R} .
\end{aligned}
$$

So, $\mathcal{S}_{2}(\mathfrak{U}, \mathfrak{H})=1$.
Conversely, consider $\mathcal{S}_{2}(\mathfrak{U}, \mathfrak{T})=1$. Since $\cos (0)=1$, we infer that

$$
\begin{aligned}
& \left|\rho_{\ell \mathfrak{U}}^{+}\left(\widetilde{\varrho}_{i}\right)-\rho_{\ell \mathfrak{T}}^{+}\left(\widetilde{\varrho}_{i}\right)\right|=0, \\
& \left|\rho_{u \mathfrak{U}}^{+}\left(\widetilde{\varrho}_{i}\right)-\rho_{u \mathfrak{H}}^{+}\left(\widetilde{\varrho}_{i}\right)\right|=0 \text {, } \\
& \left|\rho_{\ell \mathfrak{U}}^{-}\left(\widetilde{\varrho}_{i}\right)-\rho_{\ell \mathfrak{W}}^{-}\left(\widetilde{\varrho}_{i}\right)\right|=0 \text {, } \\
& \left|\rho_{u \mathfrak{u}}^{-}\left(\widetilde{\varrho}_{i}\right)-\rho_{u \mathfrak{H}}^{-}\left(\widetilde{\varrho}_{i}\right)\right|=0 \text {, } \\
& \left|\rho_{\mathfrak{U}}^{+}\left(\widetilde{\varrho}_{i}\right)-\rho_{\mathfrak{Y}}^{+}\left(\widetilde{\varrho}_{i}\right)\right|=0 \text {, } \\
& \left|\rho_{\mathfrak{U}}^{+}\left(\widetilde{\varrho}_{i}\right)-\rho_{\mathfrak{H}}^{+}\left(\widetilde{\varrho}_{i}\right)\right|=0, \quad \text { for all } \widetilde{\varrho}_{i} \in \mathfrak{R} \text {. }
\end{aligned}
$$

This gives $\left[\rho_{\ell \mathfrak{U}}^{+}\left(\widetilde{\varrho}_{i}\right), \rho_{\mathfrak{u} \mathfrak{U}}^{+}\left(\widetilde{\varrho}_{i}\right)\right]=\left[\rho_{\ell \mathfrak{H}}^{+}\left(\widetilde{\varrho}_{i}\right), \rho_{u \mathfrak{H}}^{+}\left(\widetilde{\varrho}_{i}\right)\right]$, $\left[\rho_{\ell \mathfrak{U}}^{-}\left(\widetilde{\mathrm{Q}}_{i}\right), \rho_{u \mathfrak{U}}^{-}\left(\widetilde{\mathrm{Q}}_{i}\right)\right]=\left[\rho_{\ell \mathfrak{H}}^{-}\left(\widetilde{\mathrm{Q}}_{i}\right), \rho_{\mathfrak{u} \mathfrak{H}}^{-}\left(\widetilde{\mathrm{Q}}_{i}\right)\right], \quad \rho_{\mathfrak{U}}^{+}\left(\widetilde{\mathrm{Q}}_{i}\right)=\rho_{\mathfrak{H}}^{+}\left(\widetilde{\mathrm{Q}}_{i}\right)$, and $\rho_{\mathfrak{U}}^{-}\left(\widetilde{\varrho}_{i}\right)=\rho_{\mathfrak{H}}^{-}\left(\widetilde{\varrho}_{i}\right)$, for all $\widetilde{\varrho}_{i} \in \mathfrak{R}$. Hence, $\mathfrak{U}=\mathfrak{H}$.

Example 2. Consider the CBFSs $\mathfrak{U}$ and $\mathfrak{H}$ from the previous example. By utilizing equation (7), another cosine similarity measure between $\mathfrak{U}$ and $\mathfrak{H}$ can be computed as

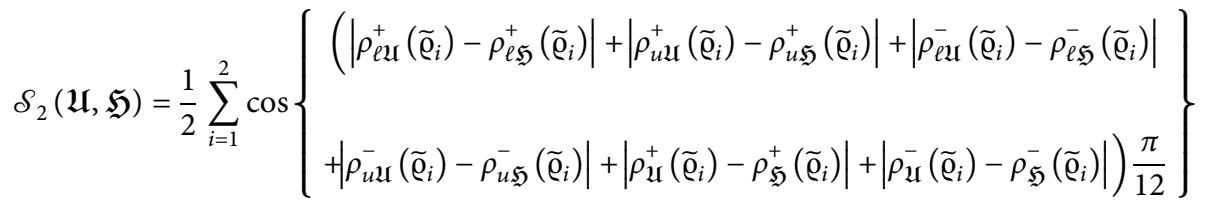

$$
\begin{aligned}
& =\frac{1}{2}\left\{\cos \left\{(|0.11-0.61|+|0.26-0.76|+|-0.38+0.59|+|-0.23+0.44|+|0.12-0.73|+|-0.31+0.41|) \frac{\pi}{12}\right\}\right. \\
& \left.+\cos \left\{(|0.22-0.59|+|0.37-0.74|+|-0.44+0.29|+|-0.29+0.14|+|0.23-0.72|+|-0.42+0.11|) \frac{\pi}{12}\right\}\right\}=0.8674 \text {. }
\end{aligned}
$$

Definition 6. Let $\left\{\left\langle\widetilde{\varrho}_{i}, \quad\left[\rho_{\ell \mathbb{I}}^{+}\left(\widetilde{\varrho}_{i}\right), \rho_{u \mathbb{U}}^{+}\left(\widetilde{\varrho}_{i}\right)\right],\left[\rho_{\ell \mathfrak{I I}}^{-}\left(\widetilde{\varrho}_{i}\right), \rho_{u \mathfrak{I I}}^{-}\right.\right.\right.$ $\left.\left.\left.\left(\widetilde{\varrho}_{i}\right)\right],\left(\rho_{\mathfrak{U}}^{+}\left(\widetilde{\varrho}_{i}\right), \rho_{\mathfrak{U}}^{-}\left(\widetilde{\varrho}_{i}\right)\right)\right\rangle: \widetilde{\varrho}_{i} \in \mathfrak{R}\right\}$ and $\mathfrak{H}=\left\{\left\langle\widetilde{\varrho}_{i},\left[\rho_{\ell \mathfrak{T}}^{+}\left(\widetilde{\varrho}_{i}\right)\right.\right.\right.$, $\left.\left.\left.\rho_{u \mathfrak{W}}^{+}\left(\widetilde{\varrho}_{i}\right)\right],\left[\rho_{\ell \mathfrak{H}}^{-}\left(\widetilde{\varrho}_{i}\right), \rho_{u \mathfrak{H}}^{-}\left(\widetilde{\varrho}_{i}\right)\right],\left(\rho_{\mathfrak{H}}^{+}\left(\widetilde{\varrho}_{i}\right), \rho_{\mathfrak{H}}^{-}\left(\widetilde{\varrho}_{i}\right)\right)\right\rangle: \quad \widetilde{\varrho}_{i} \in \mathfrak{R}\right\}$ be two CBFSs on $\Re=\left\{\widetilde{\varrho}_{1}, \widetilde{\varrho}_{2}, \ldots, \widetilde{\varrho}_{n}\right\}$; then, cosine similarity measure based on cosine function is defined as

$$
\mathcal{S}_{3}(\mathfrak{U}, \mathfrak{H})=\frac{1}{4 n(\sqrt{2}-1)} \sum_{i=1}^{n}\left\{\begin{array}{c}
{\left[\sqrt{2} \cos \left(\frac{\pi}{8}\left(\rho_{\mathfrak{\ell U}}^{+}\left(\widetilde{\varrho}_{i}\right)-\rho_{\ell \mathfrak{H}}^{+}\left(\widetilde{\varrho}_{i}\right)+\rho_{u \mathfrak{U}}^{+}\left(\widetilde{\varrho}_{i}\right)-\rho_{u \mathfrak{Y}}^{+}\left(\widetilde{\varrho}_{i}\right)\right)\right)-1\right]} \\
+\left[\sqrt{2} \cos \left(\frac{\pi}{8}\left(\rho_{\ell \mathfrak{U}}^{-}\left(\widetilde{\varrho}_{i}\right)-\rho_{\ell \mathfrak{S}}^{-}\left(\widetilde{\varrho}_{i}\right)+\rho_{u \mathfrak{U}}^{-}\left(\widetilde{\varrho}_{i}\right)-\rho_{u \mathfrak{S}}^{-}\left(\widetilde{\varrho}_{i}\right)\right)\right)-1\right] \\
+\left[\sqrt{2} \cos \left(\frac{\pi}{4}\left(\rho_{\mathfrak{U}}^{+}\left(\widetilde{\varrho}_{i}\right)-\rho_{\mathfrak{H}}^{+}\left(\widetilde{\varrho}_{i}\right)\right)\right)-1\right] \\
+\left[\sqrt{2} \cos \left(\frac{\pi}{4}\left(\rho_{\mathfrak{U}}^{-}\left(\widetilde{\varrho}_{i}\right)-\rho_{\mathfrak{H}}^{-}\left(\widetilde{\varrho}_{i}\right)\right)\right)-1\right]
\end{array}\right\} .
$$

Theorem 3. For two CBFSs $\mathfrak{U}$ and $\mathfrak{H}$, the cosine similarity measure proposed in equation (12) satisfies the following conditions: (i) $0 \leq \mathcal{S}_{3}(\mathfrak{U}, \mathfrak{W}) \leq 1$

(ii) $\mathcal{S}_{3}(\mathfrak{U}, \mathfrak{H})=\mathcal{S}_{3}(\mathfrak{H}, \mathfrak{U})$

(iii) $\mathcal{S}_{3}(\mathfrak{U}, \mathfrak{H})=1$ if $\mathfrak{U}=\mathfrak{H}$ 
Proof

(i) Let $k_{1}=\left(\rho_{\ell \mathfrak{U}}^{+}\left(\widetilde{\varrho}_{i}\right)-\rho_{\ell \mathfrak{H}}^{+}\left(\widetilde{\varrho}_{i}\right)+\rho_{u \mathfrak{U}}^{+}\left(\widetilde{\varrho}_{i}\right)-\rho_{u \mathfrak{H}}^{+}\left(\widetilde{\varrho}_{i}\right)\right) / 2$, $k_{2}=\left(\rho_{\ell \mathfrak{U}}^{-}\left(\widetilde{\varrho}_{i}\right)-\rho_{\ell \mathfrak{Y}}^{-}\left(\widetilde{\varrho}_{i}\right)+\rho_{u \mathfrak{U}}^{-}\left(\widetilde{\varrho}_{i}\right)-\rho_{u \mathfrak{H}}^{-}\left(\widetilde{\varrho}_{i}\right)\right) / 2$,

$\Rightarrow \frac{1}{\sqrt{2}} \leq \cos \left(\frac{\pi}{4} k_{\ell}\right) \leq 1$

$\Rightarrow 1 \leq \sqrt{2} \cos \left(\frac{\pi}{4} k_{\ell}\right) \leq \sqrt{2}$

$\Rightarrow 0 \leq \sqrt{2} \cos \left(\frac{\pi}{4} k_{\ell}\right)-1 \leq \sqrt{2}-1$

$\Rightarrow 0 \leq\left(\sqrt{2} \cos \left(\frac{\pi}{4} k_{1}\right)-1\right)+\left(\sqrt{2} \cos \left(\frac{\pi}{4} k_{2}\right)-1\right)+\left(\sqrt{2} \cos \left(\frac{\pi}{4} k_{3}\right)-1\right)+\left(\sqrt{2} \cos \left(\frac{\pi}{4} k_{4}\right)-1\right) \leq 4(\sqrt{2}-1)$

$\Rightarrow 0 \leq \frac{1}{4(\sqrt{2}-1)}\left[\left(\sqrt{2} \cos \left(\frac{\pi}{4} k_{1}\right)-1\right)+\left(\sqrt{2} \cos \left(\frac{\pi}{4} k_{2}\right)-1\right)+\left(\sqrt{2} \cos \left(\frac{\pi}{4} k_{3}\right)-1\right)+\left(\sqrt{2} \cos \left(\frac{\pi}{4} k_{4}\right)-1\right)\right] \leq 1$

$\Rightarrow 0 \leq \frac{1}{4 n(\sqrt{2}-1)} \sum_{i=1}^{n}\left[\left(\sqrt{2} \cos \left(\frac{\pi}{4} k_{1}\right)-1\right)+\left(\sqrt{2} \cos \left(\frac{\pi}{4} k_{2}\right)-1\right)+\left(\sqrt{2} \cos \left(\frac{\pi}{4} k_{3}\right)-1\right)+\left(\sqrt{2} \cos \left(\frac{\pi}{4} k_{4}\right)-1\right)\right] \leq 1$.

On substituting the values of $k_{1}, k_{2}, k_{3}$, and $k_{4}$, we get $0 \leq \mathcal{S}_{3}(\mathfrak{U}, \mathfrak{S}) \leq 1$.

(ii) Since cosine is an even function, $\mathcal{S}_{3}(\mathfrak{U}, \mathfrak{W})=\mathcal{S}_{3}(\mathfrak{H}, \mathfrak{U})$.

(iii) If $\mathfrak{U}=\mathfrak{H}$, then $\left[\rho_{l \mathfrak{U}}^{+}\left(\widetilde{\varrho}_{i}\right), \rho_{\mathfrak{u} \mathfrak{U}}^{+}\left(\widetilde{\varrho}_{i}\right)\right]=\left[\rho_{\ell \mathfrak{H}}^{+}\left(\widetilde{\varrho}_{i}\right)\right.$, $\left.\rho_{u \mathfrak{H}}^{+}\left(\widetilde{\varrho}_{i}\right)\right], \quad\left[\rho_{\ell \mathfrak{U}}^{-}\left(\widetilde{\varrho}_{i}\right), \rho_{u \mathfrak{U}}^{-}\left(\widetilde{\varrho}_{i}\right)\right]=\left[\rho_{\ell \mathfrak{H}}^{-}\left(\widetilde{\varrho}_{i}\right), \rho_{u \mathfrak{H}}^{-}\left(\widetilde{\varrho}_{i}\right)\right]$, $\rho_{\mathfrak{U}}^{+}\left(\widetilde{\varrho}_{i}\right)=\rho_{\mathfrak{H}}^{+}\left(\widetilde{\varrho}_{i}\right)$, and $\rho_{\mathfrak{U}}^{-}\left(\widetilde{\varrho}_{i}\right)=\rho_{\mathfrak{H}}^{-}\left(\widetilde{\varrho}_{i}\right)$, for all
$k_{3}=\rho_{\mathfrak{U}}^{+}\left(\widetilde{\varrho}_{i}\right)-\rho_{\mathfrak{H}}^{+}\left(\widetilde{\varrho}_{i}\right)$, and $k_{4}=\rho_{\mathfrak{U}}^{-}\left(\widetilde{\varrho}_{i}\right)-\rho_{\mathfrak{H}}^{-}\left(\widetilde{\varrho}_{i}\right)$. We see that $-1 \leq k_{\ell} \leq 1, \ell=1,2,3,4$. $\widetilde{\varrho}_{i} \in \mathfrak{R}$. Hence, one can easily infer that $\mathcal{S}_{3}(\mathfrak{U}, \mathfrak{S})=1$.

Example 3. Consider the CBFSs $\mathfrak{U}$ and $\mathfrak{H}$ from Example 1. between $\mathfrak{U}$ and $\mathfrak{H}$ given by Then, by utilizing equation (12), we calculate cosine SM

$$
\begin{aligned}
& \mathcal{S}_{3}(\mathfrak{U}, \mathfrak{H})=\frac{1}{4(2)(\sqrt{2}-1)} \sum_{i=1}^{2}\left\{\begin{array}{c}
{\left[\sqrt{2} \cos \left(\frac{\pi}{8}\left(\rho_{\ell \mathfrak{U}}^{+}\left(\widetilde{\varrho}_{i}\right)-\rho_{\ell \mathfrak{H}}^{+}\left(\widetilde{\varrho}_{i}\right)+\rho_{u \mathfrak{U}}^{+}\left(\widetilde{\varrho}_{i}\right)-\rho_{u \mathfrak{H}}^{+}\left(\widetilde{\varrho}_{i}\right)\right)\right)-1\right]} \\
+\left[\sqrt{2} \cos \left(\frac{\pi}{8}\left(\rho_{\ell \mathfrak{U}}^{-}\left(\widetilde{\varrho}_{i}\right)-\rho_{\ell \mathfrak{H}}^{-}\left(\widetilde{\varrho}_{i}\right)+\rho_{u \mathfrak{U}}^{-}\left(\widetilde{\varrho}_{i}\right)-\rho_{u \mathfrak{H}}^{-}\left(\widetilde{\varrho}_{i}\right)\right)\right)-1\right] \\
+\left[\sqrt{2} \cos \left(\frac{\pi}{4}\left(\rho_{\mathfrak{U}}^{+}\left(\widetilde{\varrho}_{i}\right)-\rho_{\mathfrak{H}}^{+}\left(\widetilde{\varrho}_{i}\right)\right)\right)-1\right] \\
+\left[\sqrt{2} \cos \left(\frac{\pi}{4}\left(\rho_{\mathfrak{U}}^{-}\left(\widetilde{\varrho}_{i}\right)-\rho_{\mathfrak{H}}^{-}\left(\widetilde{\varrho}_{i}\right)\right)\right)-1\right]
\end{array}\right\} \\
& =\frac{1}{8(\sqrt{2}-1)}\left\{\left[\sqrt{2} \cos \left(\frac{\pi}{8}(0.11-0.61+0.26-0.76)\right)-1\right]+\left[\sqrt{2} \cos \left(\frac{\pi}{8}(-0.38+0.59-0.23+0.44)\right)-1\right]\right. \\
& +\left[\sqrt{2} \cos \left(\frac{\pi}{4}(0.12-0.73)\right)-1\right]+\left[\sqrt{2} \cos \left(\frac{\pi}{8}(-0.31+0.41)\right)-1\right]+\left[\sqrt{2} \cos \left(\frac{\pi}{8}(0.22-0.59+0.37-0.74)\right)-1\right] \\
& +\left[\sqrt{2} \cos \left(\frac{\pi}{8}(-0.44+0.29-0.29+0.14)\right)-1\right]+\left[\sqrt{2} \cos \left(\frac{\pi}{4}(0.23-0.72)\right)-1\right] \\
& \left.+\left[\sqrt{2} \cos \left(\frac{\pi}{8}(-0.42+0.11)\right)-1\right]\right\} \\
& =0.8477 \text {. }
\end{aligned}
$$


If the weights of the elements $\widetilde{\varrho}_{i} \in \mathfrak{R},(i=1,2, \ldots, n)$ are taken into consideration, then we must use weighted versions of the above-defined cosine similarity measures as follows.
Definition 7. Let $\mathfrak{R}=\left\{\widetilde{\varrho}_{1}, \widetilde{\varrho}_{2}, \ldots, \widetilde{\varrho}_{n}\right\}$ be the universe of discourse, and let $\psi=\left\{\psi_{1}, \psi_{2}, \ldots, \psi_{n}\right\}$ be the weight vector of $\tilde{\varrho}_{i}(i=1,2, \ldots, n)$ such that $\psi_{i} \geq 0$ and $\sum_{i=1}^{n} \psi_{i}=1$. Then, for CBFSs $\mathcal{U}$ and $\mathfrak{H}$ on $\mathfrak{R}$, the weighted versions of the proposed cosine similarity measures can be defined as

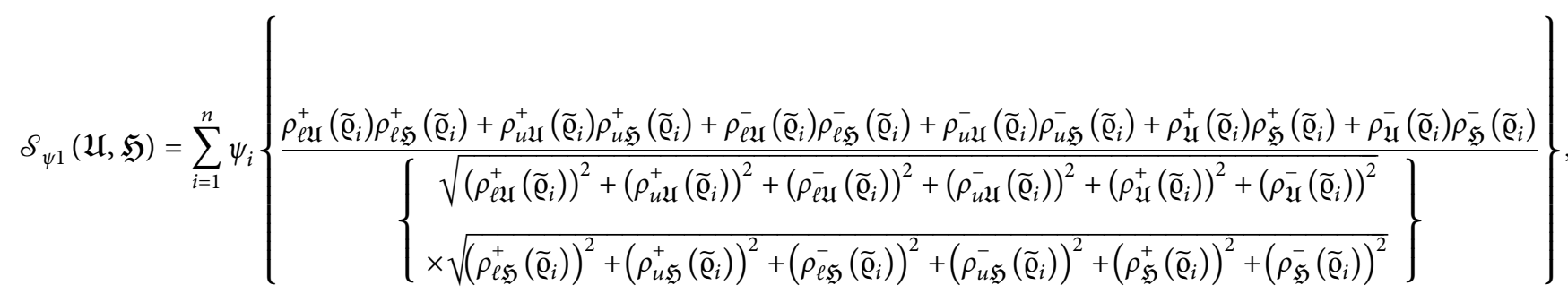

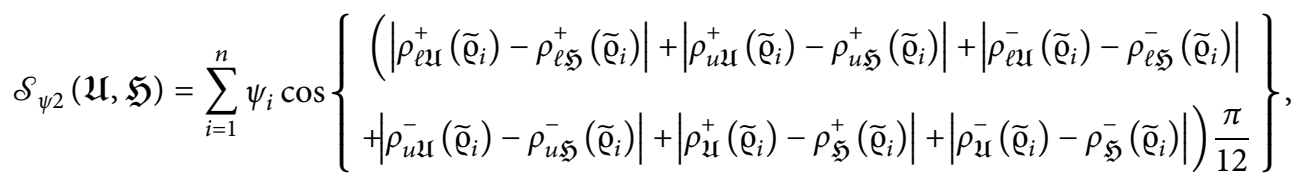

$\mathcal{S}_{\psi 3}(\mathfrak{U}, \mathfrak{H})=\frac{1}{4(\sqrt{2}-1)} \sum_{i=1}^{n} \psi_{i}\left\{\begin{array}{c}{\left[\sqrt{2} \cos \left(\frac{\pi}{8}\left(\rho_{\ell \mathfrak{U}}^{+}\left(\widetilde{\varrho}_{i}\right)-\rho_{\ell \mathfrak{W}}^{+}\left(\widetilde{\varrho}_{i}\right)+\rho_{u \mathfrak{U}}^{+}\left(\widetilde{\varrho}_{i}\right)-\rho_{u \mathfrak{H}}^{+}\left(\widetilde{\varrho}_{i}\right)\right)\right)-1\right]} \\ +\left[\sqrt{2} \cos \left(\frac{\pi}{8}\left(\rho_{\mathfrak{\ell} \mathfrak{U}}^{-}\left(\widetilde{\varrho}_{i}\right)-\rho_{\ell \mathfrak{H}}^{-}\left(\widetilde{\varrho}_{i}\right)+\rho_{u \mathfrak{U}}^{-}\left(\widetilde{\varrho}_{i}\right)-\rho_{u \mathfrak{H}}^{-}\left(\widetilde{\varrho}_{i}\right)\right)\right)-1\right] \\ +\left[\sqrt{2} \cos \left(\frac{\pi}{4}\left(\rho_{\mathfrak{U}}^{+}\left(\widetilde{\varrho}_{i}\right)-\rho_{\mathfrak{S}}^{+}\left(\widetilde{\varrho}_{i}\right)\right)\right)-1\right] \\ +\left[\sqrt{2} \cos \left(\frac{\pi}{4}\left(\rho_{\mathfrak{U}}^{-}\left(\widetilde{\varrho}_{i}\right)-\rho_{\mathfrak{Y}}^{-}\left(\widetilde{\varrho}_{i}\right)\right)\right)-1\right]\end{array}\right\}$.

Theorem 4. The weighted cosine similarity measures $\mathcal{S}_{\psi x}(\mathfrak{U}, \mathfrak{H})(x=1,2,3)$ satisfy the following axioms:

(i) $0 \leq \mathcal{S}_{\psi x}(\mathfrak{U}, \mathfrak{H}) \leq 1$

(ii) $\mathcal{S}_{\psi x}(\mathfrak{U}, \mathfrak{h})=\mathcal{S}_{\psi x}(\mathfrak{H}, \mathfrak{U})$

(iii) $\mathcal{S}_{\psi x}(\mathfrak{U}, \mathfrak{H})=1$

$\mathfrak{U}=\mathfrak{H}\left(\right.$ converse also holds in case of $\left.\mathcal{S}_{\psi 2}(\mathfrak{U}, \mathfrak{H})\right)$

Proof. The proof follows by using Theorems 1-3, respectively. Therefore, we exclude them here.

Remark 1. If $\psi=\{(1 / n),(1 / n), \ldots,(1 / n)\}$, then weighted cosine similarity measures given in equations (15)-(17) reduce to unweighted cosine similarity measures given in equations (3)-(12), respectively.

\section{Application of Cosine Similarity Measures}

Pattern recognition approaches employ machine learning and computational intelligence algorithms to identify regularities in existing patterns and to discover a pattern that is compatible with uncertain and partial data. In this section, the proposed cosine SMs are applied for the analysis of pattern recognition problem. In order to identify an unknown pattern into one of the known patterns under CBFSs, we adopt the following steps in Algorithm 1.

4.1. Illustrative Example. Bacteria recognition is an important task in microbiology. Bacteria are mainly divided into three categories on the basis of their shapes (round shape, spiral shape, and cylindrical shape). Round-shaped bacteria are called cocci, spiral-shaped bacteria are called spirilla, and cylindrical-shaped bacteria are called bacilli (https:// microbiologyinfo.com). Figure 1 demonstrates three types of bacteria.

Another way to classify bacteria is to investigate their behavior towards Gram staining test. A Gram stain is purple in color. When the stain homogenizes with bacteria in the sample, the bacteria will be pink or purple. If they remain purple, they are Gram-positive. If they are pink, they are Gram-negative (https://medlineplus.gov). Now, consider a bacterial collection consisting of Salmonella, Escherichia coli, and Shigella. All these intestinal bacteria belong to bacilli, and they are Gram-negative. The main features of these bacteria are described by

$$
\left.\Re=\left\{\widetilde{\varrho}_{1} \text { (size), } \widetilde{\varrho}_{2} \text { (flagellum), } \widetilde{\varrho}_{3} \text { (colony size }\right)\right\} .
$$




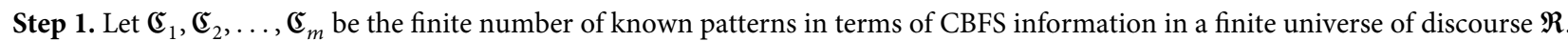
Step 2. Consider unknown pattern $\mathfrak{D}$ in the form of CBFS in $\mathfrak{R}$; this pattern is to be recognized.

Step 3. Compute the proposed cosine similarity measure between $\mathfrak{D}$ and $\mathfrak{C}_{j}(j=1,2, \ldots, m)$ by using equations (3)-(12). If the elements of the universe of discourse $\mathfrak{R}$ carry some weights, then weighted similarity measures given in equations (15)-(17) can be utilized.

Step 4. The pattern $\mathfrak{D}$ having maximum similarity measure with finite pattern $\mathfrak{C}_{j}$ is the required pattern.

Algorithm 1: Pattern recognition based on CBF information.

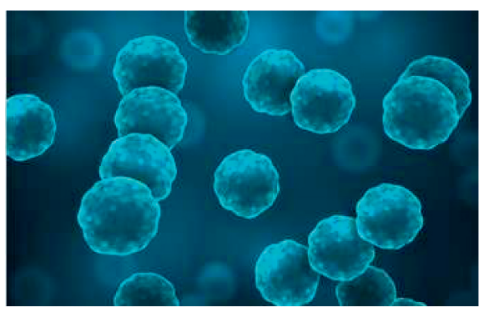

(a)

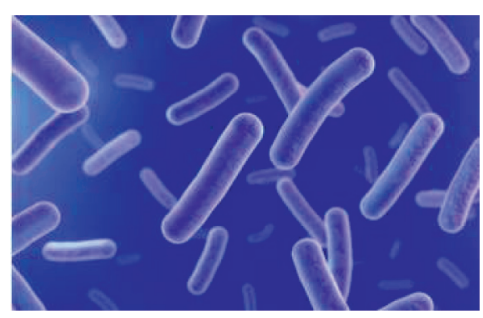

(b)

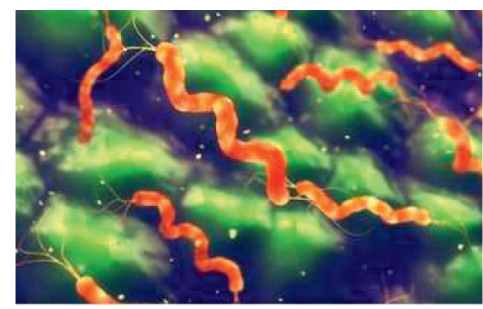

(c)

Figure 1: Three types of bacteria. (a) Cocci. (b) Bacilli. (c) Spirilla.

Suppose that a team of microbiologists assesses the presence of above features in the three bacteria and give their evaluation in the form of CBFSs.

$$
\begin{array}{r}
\mathfrak{S}_{1}(\text { Salmonella })=\left\{\begin{array}{c}
\left\langle\widetilde{\varrho}_{1},[0.61,0.72],[-0.46,-0.31],(0.52,-0.42)\right\rangle, \\
\left\langle\widetilde{\varrho}_{2},[0.82,0.93],[-0.23,-0.17],(0.73,-0.33)\right\rangle, \\
\left\langle\widetilde{\varrho}_{3},[0.47,0.58],[-0.53,-0.48],(0.36,-0.28)\right\rangle,
\end{array}\right\} \\
\mathfrak{c}_{2} \text { (E. coli) }=\left\{\begin{array}{c}
\left\langle\widetilde{\varrho}_{1},[0.34,0.49],[-0.64,-0.51],(0.29,-0.67)\right\rangle, \\
\left\langle\widetilde{\varrho}_{2},[0.79,0.88],[-0.25,-0.18],(0.76,-0.27)\right\rangle, \\
\left\langle\widetilde{\varrho}_{3},[0.62,0.77],[-0.37,-0.28],(0.49,-0.57)\right\rangle,
\end{array}\right\} \\
\mathfrak{S}_{3} \text { (Shigella) }=\left\{\begin{array}{c}
\left\langle\widetilde{\varrho}_{1},[0.57,0.69],[-0.32,-0.27],(0.47,-0.36)\right\rangle, \\
\left\langle\widetilde{\varrho}_{2},[0.19,0.28],[-0.67,-0.42],(0.22,-0.57)\right\rangle, \\
\left\langle\widetilde{\varrho}_{3},[0.53,0.68],[-0.44,-0.31],(0.51,-0.46)\right\rangle .
\end{array}\right\}
\end{array}
$$

Here, positive and negative MGs for each feature indicate the degree of existence and nonexistence of that feature in a certain bacterium. The aim of the team of microbiologists is to identify the unknown bacteria which is given as follows:

$$
\mathfrak{D}=\left\{\begin{array}{c}
\left\langle\widetilde{\varrho}_{1},[0.48,0.61],[-0.36,-0.27],(0.39,-0.27)\right\rangle, \\
\left\langle\widetilde{\varrho}_{2},[0.36,0.48],[-0.59,-0.42],(0.41,-0.57)\right\rangle, \\
\left\langle\widetilde{\varrho}_{3},[0.73,0.87],[-0.29,-0.11],(0.65,-0.32)\right\rangle .
\end{array}\right\}
$$

The team wants to know to which bacteria does $\mathfrak{D}$ belong. For such objective, we compute cosine similarity measures by utilizing equations (3)-(12). The results are summarized in Table 1.
It is obvious that the unknown bacteria belong to Shigella $\mathfrak{c}_{3}$. It is noteworthy that the computations obtained from all three cosine similarity measures are compatible with one another. Now, suppose that a weight vector $\psi=\{0.39,0.37,0.24\}$ is assigned to the elements of $\mathfrak{R}$; then, we calculate weighted cosine similarity measures by using equations (15)-(17). The computations are expressed in Table 2.

The unknown bacteria are again classified into Shigella $\mathfrak{r}_{3}$.

\section{Extended TOPSIS Method Based on Cosine Similarity Measures}

In this section, we propose the TOPSIS method based on cosine similarity measures to deal with MCGDM problems under cubic bipolar fuzzy information. Linguistic variables and terms are expressed in Table 3. 
TABLE 1: Cosine similarity measures between unknown and known patterns.

\begin{tabular}{lccc}
\hline & $\mathcal{S}\left(\mathfrak{S}_{1}, \mathfrak{D}\right)$ & $\mathcal{S}\left(\mathfrak{C}_{2}, \mathfrak{D}\right)$ & $\mathcal{S}\left(\mathfrak{C}_{3}, \mathfrak{D}\right)$ \\
\hline $\mathcal{S}_{1}$ & 0.8976 & 0.8868 & 0.9701 \\
$\mathcal{S}_{2}$ & 0.9218 & 0.9256 & 0.9819 \\
$\mathcal{S}_{3}$ & 0.9326 & 0.9268 & 0.9823 \\
\hline
\end{tabular}

TABLE 2: Weighted cosine similarity measures between unknown and known patterns.

\begin{tabular}{lccc}
\hline & $\mathcal{S}_{\psi}\left(\mathfrak{C}_{1}, \mathfrak{D}\right)$ & $\mathcal{S}_{\psi}\left(\mathfrak{C}_{2}, \mathfrak{D}\right)$ & $\mathcal{S}_{\psi}\left(\mathfrak{C}_{3}, \mathfrak{D}\right)$ \\
\hline $\mathcal{S}_{\psi 1}$ & 0.9015 & 0.8772 & 0.9726 \\
$\mathcal{S}_{\psi 2}$ & 0.9227 & 0.9196 & 0.9844 \\
$\mathcal{S}_{\psi 3}$ & 0.9335 & 0.9214 & 0.9839 \\
\hline
\end{tabular}

TABLE 3: Linguistic terms for the assessment of criteria.

\begin{tabular}{lc}
\hline Linguistic terms & Fuzzy weights \\
\hline Very important (VI) & 0.95 \\
Important (I) & 0.80 \\
Medium (M) & 0.50 \\
Less important (LI) & 0.40 \\
\hline
\end{tabular}

Figure 2 displays the flowchart diagram of Algorithm 2.

5.1. Case Study. Plastics are one of the most widely used materials in the world. The term "plastic" is derived from a Greek word "plastikos" which means fit for being shaped or molded. Plastic can embrace any shape or form. This is the reason it is utilized for a wide assortment of uses, from ordinary single-use items like bottles, boxes, and packaging to items like electronics, furniture, building materials, clothes, and food. Plastics have supplanted a broader scope of conventional materials including glass, wood, steel, and surprisingly concrete. Plastic is flexible, lightweight, moisture-resistant, and cost-friendly. These are the alluring attributes which lead to huge consumption of plastic worldwide. Due to this reason, the global plastic production has drastically increased from some 1.5 million metric tons in 1950 to 368 million metric tons in 2019. China is the world's largest manufacturer of plastic, accounting for 31 percent of global plastic production (https:// www.statista.com/statistics/282 732/global-production-ofplastics-since-1950/). Figure 3 shows the expansion in global plastic production from 1950 to 2019.

However, owing to the durability and very slow degradation of plastics, the problem arises in the life management of the goods made from them. Plastics account for more than 12 percent of all solid wastes discharged (https://datatopics. worldbank.org). Figure 4 shows how much time different plastic items take to degrade. Most of the plastic waste has been dumped in the landfills or in the wild. Since plastics take too much time to decay, so throwing them into landfills just clogs up the valuable landfill space. About 8 million metric tons of plastic garbage is washed into the oceans by rivers around the world [49] every year. This has severe environmental consequences on marine life and human health.

Plastic recycling is the best way to manage plastic waste. Recycling of plastics can be defined as a process which involves collection, separation, and processing of plastic wastes to form useful products. Plastic recycling has several advantages. Recycling one ton amount of plastics can conserve 7.4 cubic yards of landfill space (https://www. online-sciences.com). It also helps to reduce water pollution. Since plastics are made up of fossil fuels (crude oil, natural gas, etc.) or renewables (sugar cane, starch, etc.), recycling of plastics reduces the consumption of these natural resources resulting in their conservation.

There are three methods of plastic recycling.

5.1.1. Mechanical Recycling. The physical method of material reprocessing of plastic wastes into plastic products is known as mechanical recycling. First of all, the plastics are categorized by their resin type. Then, plastic recyclables are shredded. Impurities such as paper labels are subsequently removed from the shredded particles. This substance is melted and frequently extruded into pellets which are then utilized to manufacture other products.

5.1.2. Chemical Recycling. Chemical recycling is based on the principle of breaking down a polymeric product into its individual components (monomers for plastics), which can then be used as input raw material to recreate the original product or others.

5.1.3. Energy Recovery. Waste-to-energy technology is known as energy recovery. Although the process of combustion and gasification is similar on a fundamental level, cremation provides the vitality of high-temperature heat. Waste materials are incinerated, which produces ash, flue gas, and heat. The heat created by incineration can be used to create electricity in some instances.

Plastics life cycle is demonstrated in Figure 5.

Now-a-days, the industries which manufacture plastic products are keen to recycle their waste materials. The key issue is determining which recycling method to use. Selecting the best plastic recycling method is a MCGDM problem. To select the best plastic recycling method, the criteria under consideration are given in Table 4.

5.2. Numerical Illustration. Plastic materials are widely used during the manufacturing of electronic devices. Therefore, it is necessary for the electronics manufacturing companies to manage plastic waste:

Step 1. Suppose that a well-known electronic devices manufacturing company wants to manage plastic waste from end-of-life electronics. Let $\mathscr{A}=\left\{\mathscr{A}_{1}, \mathscr{A}_{2}, \mathscr{A}_{3}\right\}$ be the set of alternatives, where $\mathscr{A}_{1}=$ mechanical recycling, $\mathscr{A}_{2}=$ chemical recycling, and $\mathscr{A}_{3}=$ energy recovery. To choose the best alternative, the set of criteria is given in Table 4 and let the set of decision makers be $\mathscr{E}=\left\{\mathscr{E}_{1}, \mathscr{E}_{2}, \mathscr{E}_{3}, \mathscr{E}_{4}\right\}$.

Step 2. The weighted criterion matrix $\mathscr{L}$ is constructed on the basis of linguistic variables given in Table 3 as follows: 


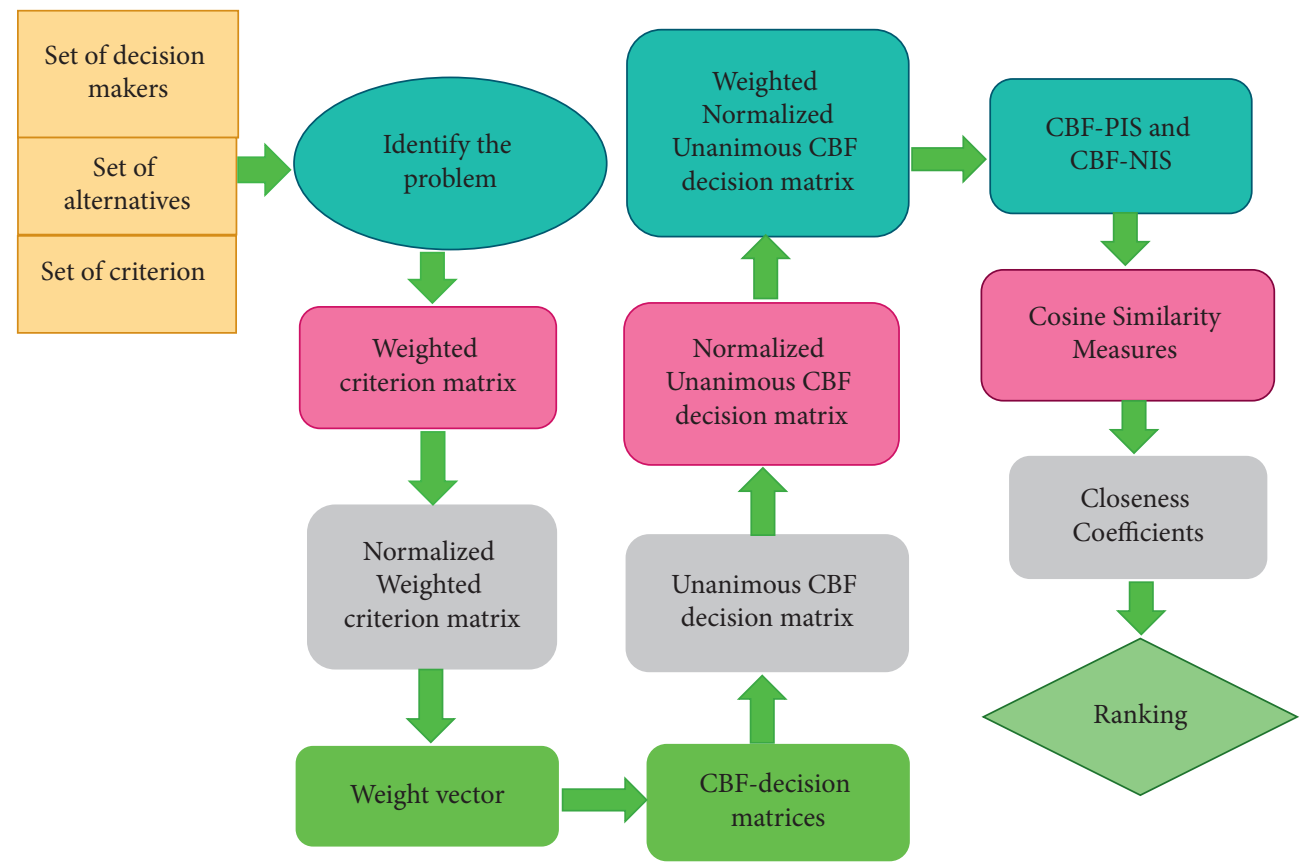

FIGURE 2: Flowchart diagram of Algorithm 2.

$$
\begin{aligned}
& \mathscr{L}=\left[\varrho_{i k}\right]_{4 \times 4}=\left(\begin{array}{cccc}
\mathrm{VI} & \mathrm{I} & \mathrm{I} & \mathrm{M} \\
\mathrm{I} & \mathrm{M} & \mathrm{VI} & \mathrm{I} \\
\mathrm{VI} & \mathrm{I} & \mathrm{M} & \mathrm{LI} \\
\mathrm{M} & \mathrm{VI} & \mathrm{I} & \mathrm{M}
\end{array}\right), \\
& \mathscr{L}=\left[\varrho_{i k}\right]_{4 \times 4}=\left(\begin{array}{cccc}
0.95 & 0.80 & 0.80 & 0.50 \\
0.80 & 0.50 & 0.95 & 0.80 \\
0.95 & 0.80 & 0.50 & 0.40 \\
0.50 & 0.95 & .80 & 0.50
\end{array}\right) .
\end{aligned}
$$

Step 3. The normalized weighted criterion matrix $\mathscr{M}$ is obtained by using Algorithm 2 as follows:

$$
\mathscr{M}=\left[\psi_{i k}\right]_{4 \times 4}=\left(\begin{array}{cccc}
0.5787 & 0.5129 & 0.5129 & 0.4385 \\
0.4873 & 0.3206 & 0.6091 & 0.7016 \\
0.5787 & 0.5129 & 0.3206 & 0.3508 \\
0.3046 & 0.6091 & 0.5129 & 0.4385
\end{array}\right) \text {. }
$$

Step 4. By using Algorithm 2, the weight vector is given by $\mathscr{W}=\{0.2502,0.2510,0.2510,0.2477\}$.

Step 5. The CBF decision matrices $\mathbb{Q}_{i}$ are provided by the decision makers in which each row represents an alternative and each column represents a criterion. The unanimous CBF decision matrix $T$ is obtained by using Algorithm 2.

Step 6. Since cost, $\mathrm{CO}_{2}$ emissions, and energy consumption are cost-type criteria, we construct normalized unanimous CBF decision matrix $\widehat{T}$ by utilizing Algorithm 2.

Step 7. By using Algorithm 2, the weighted normalized CBF decision matrix $Y$ is obtained.

Step 8. The CBF-PIS and CBF-NIS are obtained by using Algorithm 2:

$$
\begin{aligned}
\mathrm{CBF}-\mathrm{PIS}=\left\{\begin{array}{l}
\langle[0.2150,0.2663],[-0.9573,-0.9243],(0.1904,-0.9113)\rangle, \\
\langle[0.2735,0.3590],[-0.8760,-0.8138],(0.2869,-0.9077)\rangle, \\
\langle[0.2429,0.3409],[-0.9044,-0.8486],(0.1957,-0.9077)\rangle, \\
\langle[0.2080,0.3051],[-0.9661,-0.9250],(0.2236,-0.9715)\rangle,
\end{array}\right\} \\
\mathrm{CBF}-\mathrm{NIS}=\left\{\begin{array}{l}
\langle[0.0102,0.0514],[-0.9113,-0.8763],(0.0573,-0.8365)\rangle, \\
\langle[0.0344,0.0666],[-0.8361,-0.7791],(0.0791,-0.8138)\rangle, \\
\langle[0.0515,0.0791],[-0.7791,-0.7131],(0.0760,-0.7453)\rangle, \\
\langle[0.0480,0.0813],[-0.9218,-0.8953],(0.1081,-0.8918)\rangle .
\end{array}\right\}
\end{aligned}
$$


Step 1. Identify MCGDM problem:

Suppose that $\mathscr{E}=\left\{\mathscr{E}_{i}: i=1,2, \ldots, n\right\}$ is the set of DMs, $\mathscr{A}=\left\{\mathscr{A}_{j}: j=1,2, \ldots, \ell\right\}$ is the set of alternatives, and $\mathscr{C}=\left\{\mathscr{C}_{k}: k=\right.$ $1,2, \ldots, m\}$ is the set of criterion.

Step 2. Construct the weighted criterion matrix with the help of linguistic variables that are given in Table $3 . \mathscr{L}=\left[\varrho_{i k}\right]_{n \times m}=$ $\left(\begin{array}{cccc}\varrho_{11} & \varrho_{12} & \cdots & \varrho_{1 m} \\ \varrho_{21} & \varrho_{22} & \cdots & \varrho_{2 m} \\ \vdots & \vdots & \ddots & \vdots \\ \varrho_{i 1} & \varrho_{i 2} & \cdots & \varrho_{i m} \\ \vdots & \vdots & \ddots & \vdots \\ \varrho_{n 1} & \varrho_{n 2} & \cdots & \end{array}\right.$

where $\varrho_{i k}$ is the fuzzy weight assigned by the decision maker $\mathscr{E}_{i}$ to the criterion $\mathscr{C}_{k}$ by considering the values of linguistic variables.

Step 3. Obtain normalized weighted criterion matrix $\mathscr{M}=\left[\psi_{i k}\right]_{n \times m}=\left(\begin{array}{cccc}\psi_{11} & \psi_{12} & \cdots & \psi_{1 m} \\ \psi_{21} & \psi_{22} & \cdots & \psi_{2 m} \\ \vdots & \vdots & \ddots & \vdots \\ \psi_{i 1} & \psi_{i 2} & \cdots & \psi_{i m} \\ \vdots & \vdots & \ddots & \vdots \\ \psi_{n 1} & \psi_{n 2} & \cdots & \psi_{n m}\end{array}\right)$,
$\quad$ where $\psi_{i k}=\varrho_{i k} / \sqrt{\sum_{i=1}^{n} \varrho_{i k}^{2}}$.

Step 4. Construct weight vector $\mathscr{W}=\left\{w_{1}, w_{2}, \ldots, w_{m}\right\}$ as follows: $w_{k}=\left(w_{k} / \sum_{k=1}^{m} w_{k}\right)$, where $w_{k}=(1 / n) \sum_{i=1}^{n} \psi_{i k}$.

Step 5. Construct CBF-decision matrices $Q_{i}=\left[q_{j k}^{i}\right]_{l \times m}=\left(\begin{array}{cccc}q_{11}^{i} & q_{12}^{i} & \cdots & q_{1 m}^{i} \\ q_{21} & q_{22}^{i} & \cdots & q_{2 m}^{i} \\ \vdots & \vdots & \ddots & \vdots \\ i & i & \cdots & q_{j m}^{i} \\ q_{j 1} & q_{j 2} & \cdots & q_{j m} \\ \vdots & \vdots & \ddots & \vdots \\ q_{l 1} & q_{l 2}^{i} & \cdots & q_{l m}^{i}\end{array}\right)$,

where $q_{j k}^{i}$ is a CBFS element which is assigned by the decision maker $\mathscr{E}_{i}$. The unanimous CBF decision matrix can be obtained as follows $T=\left[t_{j k}\right]_{l \times m}=\left(\begin{array}{cccc}t_{11} & t_{12} & \cdots & t_{1 m} \\ t_{21} & t_{22} & \cdots & t_{2 m} \\ \vdots & \vdots & \ddots & \vdots \\ t_{j 1} & t_{j 2} & \cdots & t_{j m} \\ \vdots & \vdots & \ddots & \vdots \\ t_{l 1} & t_{l 2} & \cdots & t_{l m}\end{array}\right)$,

where $t_{j k}=\cup_{i=1}^{n} q_{j k}^{i}=\left\langle\max _{1 \leq i \leq n}\left[\rho_{\ell q_{j k}^{+}}^{+}, \rho_{u q_{j k}^{i}}^{+}\right], \min _{1 \leq i \leq n}\left[\rho_{\ell q_{j k}^{i}}^{-}, \rho_{u q_{j k}^{i}}^{-}\right],\left(\max _{1 \leq i \leq n} \rho_{q_{j k}^{i}}^{+}, \min _{1 \leq i \leq n} \rho_{q_{j k}^{i}}^{-}\right)\right\rangle$.

Step 6. In many decision-making problems, the criteria under consideration are of two types, i.e., cost and benefit. Therefore, we need

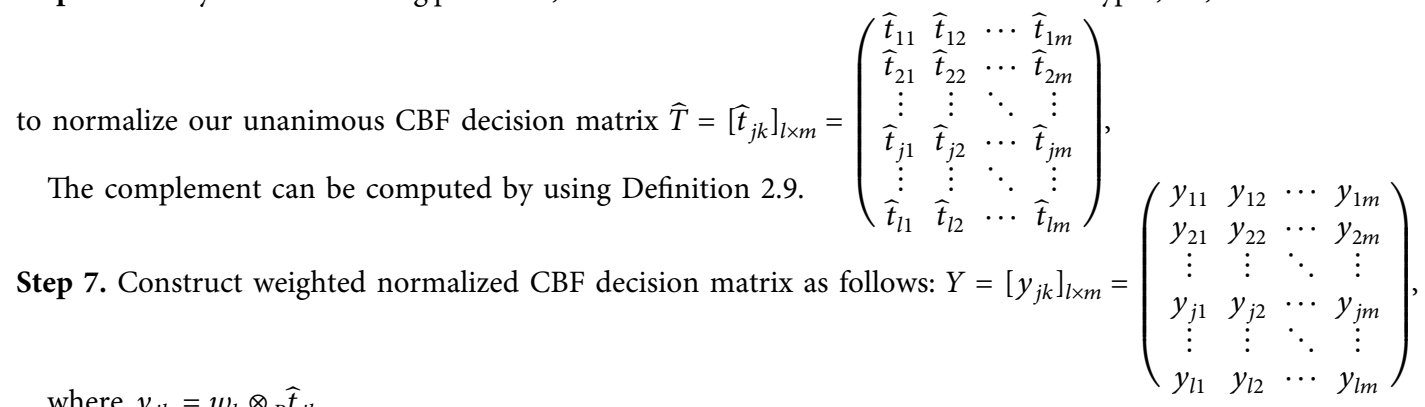

Step 8. Obtain the CBF-PIS and CBF-NIS given by the following formulas: CBF - PIS $=\left\{y_{k}^{p}: k=1,2 \ldots, m\right\}=$ $\left\{\cup_{j=1}^{\ell} y_{j k}: k=1,2, \ldots, m\right\}=\left\{\left\langle\max _{1 \leq j \leq \ell}\left[\rho_{\ell y_{j k}}^{+}, \rho_{u y_{j k}}^{+}\right], \min _{1 \leq j \leq \ell}\left[\rho_{\ell y_{j k}}^{-}, \rho_{u y_{j k}}^{-}\right],\left(\max _{1 \leq j \leq \ell} \rho_{y_{j k}}^{+}, \min _{1 \leq j \leq \ell} \rho_{y_{j k}}^{-}\right)\right\rangle: k=1,2, \ldots, m\right\}$,

$\mathrm{CBF}-\mathrm{NIS}=\left\{y_{k}^{n}: k=1,2 \ldots, m\right\}=\left\{\cap_{j=1}^{\ell} y_{j k}: k=1,2, \ldots, m\right\}=\left\{\left\langle\min _{1 \leq j \leq \ell}\left[\rho_{\ell y_{j k}}^{+}, \rho_{u y_{j k}}^{+}\right], \max _{1 \leq j \leq \ell}\left[\rho_{\ell y_{j k}}^{-}, \rho_{u y_{j k}}^{-}\right], \quad\left(\min _{1 \leq j \leq \ell} \rho_{y_{j k}}^{+}\right.\right.\right.$, $\left.\left.\left.\max _{1 \leq j \leq \ell} \rho_{y_{j k}}^{-}\right)\right\rangle: k=1,2, \ldots, m\right\}$.

Step 9. Compute the cosine similarity measure between each alternative and PIS and the cosine similarity measure between each alternative and NIS by utilizing equations (3)-(12).

If we use equation (3), then $\mathcal{S}_{1}\left(\mathscr{A}_{j}, \mathrm{CBF}-\mathrm{PIS}\right)=(1 / m) \sum_{k=1}^{m}\left\{\left(\rho_{\ell y_{j k}}^{+} \rho_{\ell y_{k}^{p}}^{+}+\rho_{u y_{j k}}^{+} \rho_{u y_{k}^{p}}^{+}+\rho_{\ell y_{j k}}^{-} \rho_{\ell y_{k}^{p}}^{-}+\rho_{u y_{j k}}^{-} \rho_{u y_{k}^{p}}^{-}+\rho_{y_{j k}}^{+} \rho_{y_{k}^{p}}^{+}+\rho_{y_{j k}}^{-} \rho_{y_{k}^{p}}^{-}\right) /\right.$ $\left\{\begin{array}{l}\sqrt{\left(\rho_{\ell y_{j k}}^{+}\right)^{2}+\left(\rho_{u y_{j k}}^{+}\right)^{2}+\left(\rho_{\ell y_{j k}}^{-}\right)^{2}+\left(\rho_{u y_{j k}}^{-}\right)^{2}+\left(\rho_{y_{j k}}^{+}\right)^{2}+\left(\rho_{y_{j k}}^{-}\right)^{2}} \\ \times \sqrt{\left(\rho_{\ell y_{k}^{p}}^{+}\right)^{2}+\left(\rho_{u y_{k}^{p}}^{+}\right)^{2}+\left(\rho_{\ell y_{k}^{p}}^{-}\right)^{2}+\left(\rho_{u y_{k}^{p}}^{-}\right)^{2}+\left(\rho_{y_{k}^{p}}^{+}\right)^{2}+\left(\rho_{y_{k}^{p}}^{-}\right)^{2}}\end{array}\right\}$, 
$\left.\begin{array}{l}\mathcal{S}_{1}\left(\mathscr{A}_{j}, \mathrm{CBF}-\mathrm{NIS}\right)=(1 / m) \sum_{k=1}^{m} \\ \left\{\begin{array}{l}\sqrt{\left(\rho_{\ell y_{j k}}^{+}\right)^{2}+\left(\rho_{u y_{j k}}^{+}\right)^{2}+\left(\rho_{\ell y_{j k}}^{-}\right)^{2}+\left(\rho_{u y_{j k}}^{-}\right)^{2}+\left(\rho_{y_{j k}}^{+}\right)^{2}+\left(\rho_{y_{j k}}^{-}\right)^{2}} \\ \times \sqrt{\left(\rho_{\ell y_{k}^{n}}^{+}\right)^{2}+\left(\rho_{u y_{k}^{n}}^{+}\right)^{2}+\left(\rho_{\ell y_{k}^{n}}^{-}\right)^{2}+\left(\rho_{u y_{k}^{n}}^{-}\right)^{2}+\left(\rho_{y_{k}^{n}}^{+}\right)^{2}+\left(\rho_{y_{k}^{n}}^{-}\right)^{2}}\end{array}\right\}\end{array}\right\} \begin{aligned} & \left\{\left(\rho_{\ell y_{j k}}^{+} \rho_{\ell y_{k}^{n}}^{+}+\rho_{u y_{j k}}^{+} \rho_{u y_{k}^{n}}^{+}+\rho_{\ell y_{j k}}^{-} \rho_{\ell y_{k}^{n}}^{-}+\rho_{u y_{j k}}^{-} \rho_{u y_{k}^{n}}^{-}+\rho_{y_{j k}}^{+} \rho_{y_{k}^{n}}^{+}+\rho_{y_{j k}}^{-} \rho_{y_{k}^{n}}^{-}\right) /\right. \\ & \end{aligned}$

If we use equation (7), then $\delta_{2}\left(\mathscr{A}_{j}, \mathrm{CBF}-\mathrm{PIS}\right)=(1 / m) \sum_{k=1}^{m} \cos \left\{\begin{array}{c}\left(\left|\rho_{\ell y_{j k}}^{+}-\rho_{\ell y_{k}^{p}}^{+}\right|+\left|\rho_{u y_{j k}}^{+}-\rho_{u y_{k}^{p}}^{+}\right|+\left|\rho_{\ell y_{j k}}^{-}-\rho_{\ell y_{k}^{p}}^{-}\right|\right. \\ \left.+\left|\rho_{u y_{j k}}^{-}-\rho_{u y_{k}^{p}}^{-}\right|+\left|\rho_{y_{j k}}^{+}-\rho_{y_{k}^{p}}^{+}\right|+\left|\rho_{y_{j k}}^{-}-\rho_{y_{k}^{p}}^{-}\right|\right)(\pi / 12)\end{array}\right\}$,

$$
\mathcal{S}_{2}\left(\mathscr{A}_{j}, \mathrm{CBF}-\mathrm{NIS}\right)=(1 / m) \sum_{k=1}^{m} \cos \left\{\begin{array}{c}
\left(\left|\rho_{\ell y_{j k}}^{+}-\rho_{\ell y_{k}^{n}}^{+}\right|+\left|\rho_{u y_{j k}}^{+}-\rho_{u y_{k}^{n}}^{+}\right|+\left|\rho_{\ell y_{j k}}^{-}-\rho_{\ell y_{k}^{n}}^{-}\right|\right. \\
\left.+\left|\rho_{u y_{j k}}^{-}-\rho_{u y_{k}^{n}}^{-}\right|+\left|\rho_{y_{j k}}^{+}-\rho_{y_{k}^{n}}^{+}\right|+\left|\rho_{y_{j k}}^{-}-\rho_{y_{k}^{n}}^{-}\right|\right)(\pi / 12)
\end{array}\right\} \text {. }
$$

If we use equation (12), then $\mathcal{S}_{3}\left(\mathscr{A}_{j}, \mathrm{CBF}-\mathrm{PIS}\right)=(1 / 4 m(\sqrt{2}-1)) \sum_{k=1}^{m}\left\{\left[\sqrt{2} \cos \left((\pi / 8)\left(\rho_{\ell y_{j k}}^{+}-\rho_{\ell y_{k}^{p}}^{+}+\rho_{u y_{j k}}^{+}-\rho_{u y_{k}^{p}}^{+}\right)\right)-1\right]+[\sqrt{2}\right.$ $\left.\left.\cos \left((\pi / 8)\left(\rho_{\ell y_{j k}}^{-}-\rho_{\ell y_{k}^{p}}^{-}+\rho_{u y_{j k}}^{-}-\rho_{u y_{k}^{p}}^{-}\right)\right)-1\right]+\left[\sqrt{2} \cos \left((\pi / 4)\left(\rho_{y_{j k}}^{+}-\rho_{y_{k}^{p}}^{+}\right)\right)-1\right]+\left[\sqrt{2} \cos \left((\pi / 4)\left(\rho_{y_{j k}}^{-}-\rho_{y_{k}^{p}}^{p}\right)\right)-1\right]\right\}$,

$\mathcal{S}_{3}\left(\mathscr{A}_{j}, \mathrm{CBF}-\mathrm{NIS}\right)=(1 / 4 m(\sqrt{2}-1)) \sum_{k=1}^{m}\left\{\left[\sqrt{2} \cos \left((\pi / 8)\left(\rho_{\ell y_{j k}}^{+}-\rho_{\ell y_{k}^{n}}^{+}+\rho_{u y_{j k}}^{+}-\rho_{u y_{k}^{n}}^{+}\right)\right)-1\right]+\left[\sqrt{2} \cos \left((\pi / 8)\left(\rho_{\ell y_{j k}}^{-}-\rho_{\ell y_{k}^{n}}^{-}+\rho_{u y_{j k}}^{-}\right.\right.\right.\right.$ $\left.\left.\left.\left.-\rho_{u y_{k}^{n}}^{-}\right)\right)-1\right]+\left[\sqrt{2} \cos \left((\pi / 4)\left(\rho_{y_{j k}}^{+}-\rho_{y_{k}^{n}}^{+}\right)\right)-1\right]+\left[\sqrt{2} \cos \left((\pi / 4)\left(\rho_{y_{j k}}^{-}-\rho_{y_{k}^{n}}^{-}\right)\right)-1\right]\right\}$.

Step 10. Calculate the value of closeness coefficient of each alternative to ideal solution by using the following:

If we use $\delta_{1}$, then $\alpha_{j}=\mathcal{S}_{1}\left(\mathscr{A}_{j}, \mathrm{CBF}-\mathrm{PIS}\right) /\left(\mathcal{S}_{1}\left(\mathscr{A}_{j}, \mathrm{CBF}-\mathrm{PIS}\right)+\mathcal{S}_{1}\left(\mathscr{A}_{j}, \mathrm{CBF}-\mathrm{NIS}\right)\right)$

If we use $\mathcal{S}_{2}$, then $\beta_{j}=\mathcal{S}_{2}\left(\mathscr{A}_{j}, \mathrm{CBF}-\mathrm{PIS}\right) /\left(\mathcal{S}_{2}\left(\mathscr{A}_{j}, \mathrm{CBF}-\mathrm{PIS}\right)+\mathcal{S}_{2}\left(\mathscr{A}_{j}, \mathrm{CBF}-\mathrm{NIS}\right)\right)$

If we use $\mathcal{S}_{3}$, then $\gamma_{j}=\mathcal{S}_{3}\left(\mathscr{A}_{j}, \mathrm{CBF}-\mathrm{PIS}\right) /\left(\mathcal{S}_{3}\left(\mathscr{A}_{j}, \mathrm{CBF}-\mathrm{PIS}\right)+\mathcal{S}_{3}\left(\mathscr{A}_{j}, \mathrm{CBF}-\mathrm{NIS}\right)\right)$

Step 11. Rank the alternatives by arranging the values of closeness coefficients in the descending order. The best alternative has the maximum value of closeness coefficient.

Algorithm 2: Extension of TOPSIS towards CBF information.

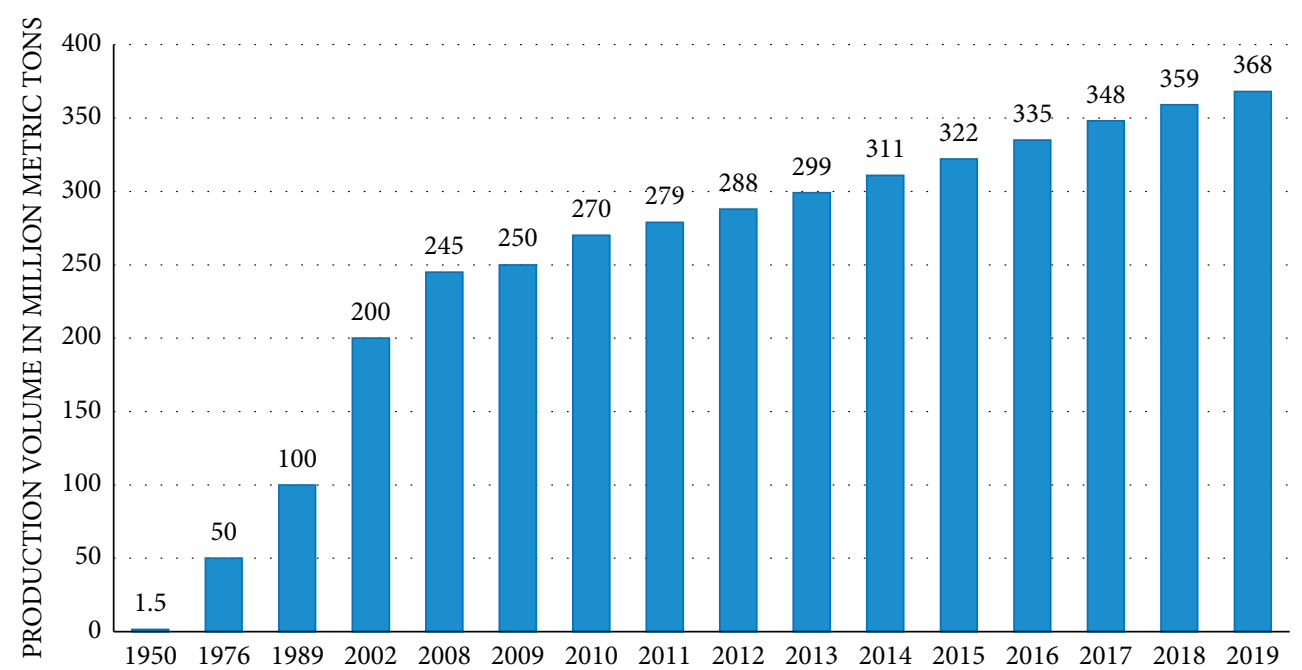

Figure 3: Global plastic production from 1950 to 2019.

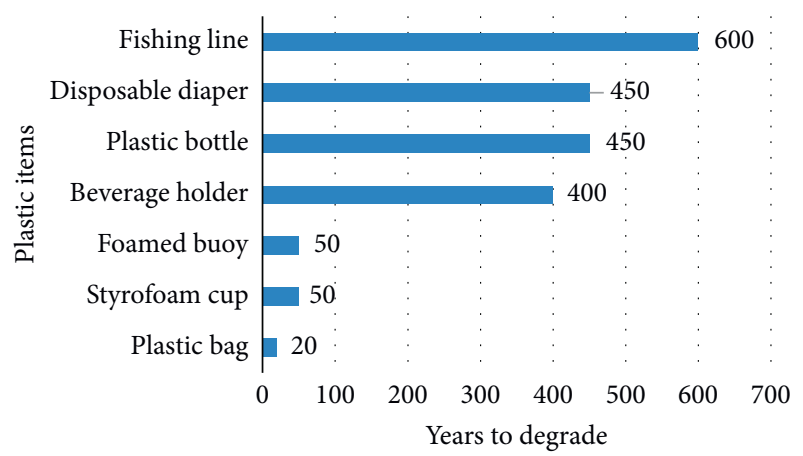

FIgURE 4: Degradation of different plastic items [48].
Step 9. Now, we compute the SMs between each alternative $\mathscr{A}_{j}$ and PIS and the similarity measure between $\mathscr{A}_{j}$ and NIS. By using equation (3), we get

$$
\begin{aligned}
& \mathcal{S}_{1}\left(\mathscr{A}_{1}, \mathrm{CBF}-\mathrm{PIS}\right)=0.9948 \\
& \mathcal{S}_{1}\left(\mathscr{A}_{2}, \mathrm{CBF}-\mathrm{PIS}\right)=0.9807 \\
& \mathcal{S}_{1}\left(\mathscr{A}_{3}, \mathrm{CBF}-\mathrm{PIS}\right)=0.9818 \\
& \mathcal{S}_{1}\left(\mathscr{A}_{1}, \mathrm{CBF}-\mathrm{NIS}\right)=0.9787 \\
& \mathcal{S}_{1}\left(\mathscr{A}_{2}, \mathrm{CBF}-\mathrm{NIS}\right)=0.9994 \\
& \mathcal{S}_{1}\left(\mathscr{A}_{3}, \mathrm{CBF}-\mathrm{NIS}\right)=0.9941 .
\end{aligned}
$$




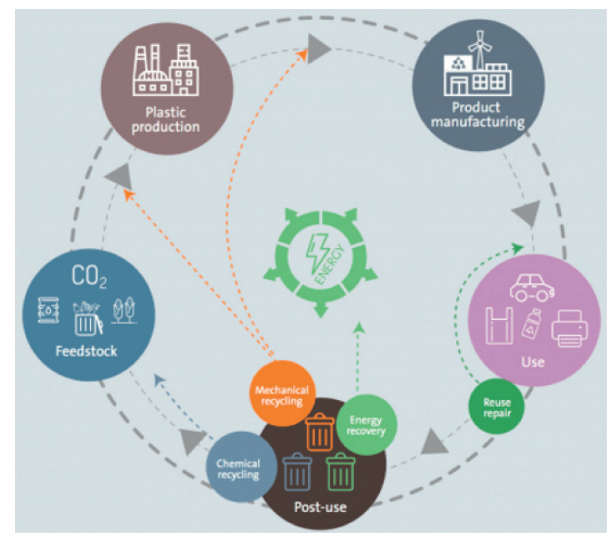

Figure 5: Plastics life cycle.

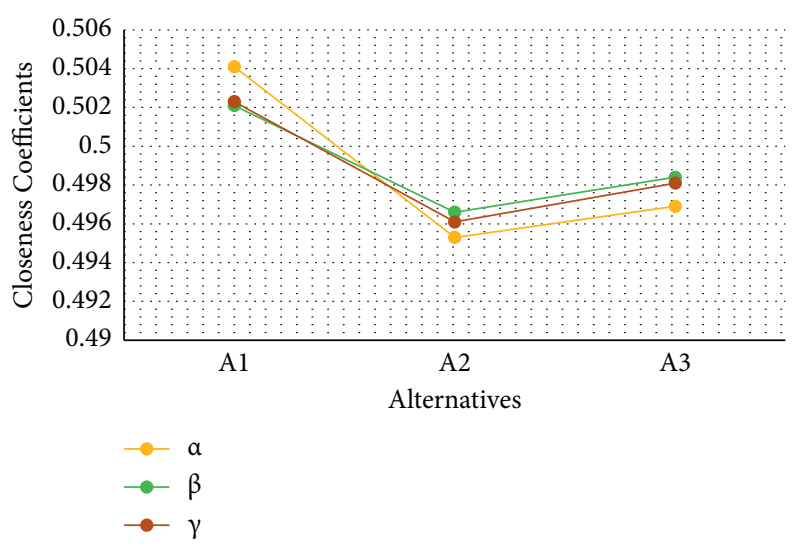

FIGURE 6: Ranking of alternatives.

TABLE 4: Criteria for the selection of best plastic recycling method.

\begin{tabular}{lc}
\hline Criteria & Description \\
\hline (i) Cost $\left(\mathscr{C}_{1}\right)$ & This includes transportation cost of \\
plastic waste, cost of plastic separation, \\
worker's salary, chemical costs \\
(ii) $\mathrm{CO}_{2}$ emissions & This is related to the $\mathrm{CO}_{2}$ emissions \\
$\left(\mathscr{C}_{2}\right)$ & during recycling process \\
(iii) Technical & This includes operating capability of \\
capability $\left(\mathscr{C}_{3}\right)$ & instruments, ingredients, and \\
(iv) Energy & configuration plan during recycling \\
consumption $\left(\mathscr{C}_{4}\right)$ & This includes the total energy required to \\
\end{tabular}

By using equation (7), we have

$$
\begin{aligned}
& \mathcal{S}_{2}\left(\mathscr{A}_{1}, \mathrm{CBF}-\mathrm{PIS}\right)=0.9956, \\
& \mathcal{S}_{2}\left(\mathscr{A}_{2}, \mathrm{CBF}-\mathrm{PIS}\right)=0.9850, \\
& \mathcal{S}_{2}\left(\mathscr{A}_{3}, \mathrm{CBF}-\mathrm{PIS}\right)=0.9892, \\
& \mathcal{S}_{2}\left(\mathscr{A}_{1}, \mathrm{CBF}-\mathrm{NIS}\right)=0.9872, \\
& \mathcal{S}_{2}\left(\mathscr{A}_{2}, \mathrm{CBF}-\mathrm{NIS}\right)=0.9985, \\
& \mathcal{S}_{2}\left(\mathscr{A}_{3}, \mathrm{CBF}-\mathrm{NIS}\right)=0.9956 .
\end{aligned}
$$

$$
\begin{aligned}
& \mathcal{S}_{3}\left(\mathscr{A}_{1}, \mathrm{CBF}-\mathrm{PIS}\right)=0.9931 \\
& \mathcal{S}_{3}\left(\mathscr{A}_{2}, \mathrm{CBF}-\mathrm{PIS}\right)=0.9829 \\
& \mathcal{S}_{3}\left(\mathscr{A}_{3}, \mathrm{CBF}-\mathrm{PIS}\right)=0.9849 \\
& \mathcal{S}_{3}\left(\mathscr{A}_{1}, \mathrm{CBF}-\mathrm{NIS}\right)=0.9840 \\
& \mathcal{S}_{3}\left(\mathscr{A}_{2}, \mathrm{CBF}-\mathrm{NIS}\right)=0.9982 \\
& \mathcal{S}_{3}\left(\mathscr{A}_{3}, \mathrm{CBF}-\mathrm{NIS}\right)=0.9923 .
\end{aligned}
$$

Step 10. The values of closeness coefficient for three different cosine similarity measures are calculated by using Algorithm 2:

$$
\begin{aligned}
& \alpha_{1}=0.5041, \\
& \alpha_{2}=0.4953, \\
& \alpha_{3}=0.4969, \\
& \beta_{1}=0.5021, \\
& \beta_{2}=0.4966, \\
& \beta_{3}=0.4984, \\
& \gamma_{1}=0.5023, \\
& \gamma_{2}=0.4961, \\
& \gamma_{3}=0.4981 .
\end{aligned}
$$

By using equation (7), we get 


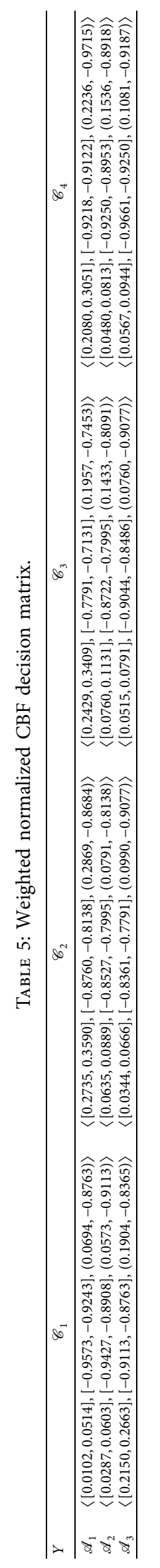


TABLE 6: Comparison of proposed Algorithm 2 with existing methods.

\begin{tabular}{lcc}
\hline Methods & Ranking & Optimal alternative \\
\hline Algorithm (Eraslan and Karaaslan [50]) & $\mathscr{A}_{1}>\mathscr{A}_{3}>\mathscr{A}_{2}$ & $\mathscr{A}_{1}$ \\
Algorithm (Mahmood et al. [10]) & $\mathscr{A}_{1}>\mathscr{A}_{3}>\mathscr{A}_{2}$ & $\mathscr{A}_{1}$ \\
Algorithm (Gundogdu and Kahraman [11]) & $\mathscr{A}_{1}>\mathscr{A}_{3}>\mathscr{A}_{2}$ & $\mathscr{A}_{1}$ \\
Algorithm (Zhang and Xu [31]) & $\mathscr{A}_{1}>\mathscr{A}_{2}>\mathscr{A}_{3}$ & $\mathscr{A}_{1}$ \\
Algorithm (Garg and Kaur [35]) & $\mathscr{A}_{1}>\mathscr{A}_{3}>\mathscr{A}_{2}$ & $\mathscr{A}_{1}$ \\
Algorithm (Tehrim and Riaz [36-38]) & $\mathscr{A}_{1}>\mathscr{A}_{2}>\mathscr{A}_{3}$ & $\mathscr{A}_{1}$ \\
Algorithm (proposed) & $\mathscr{A}_{1}>\mathscr{A}_{3}>\mathscr{A}_{2}$ & $\mathscr{A}_{1}$ \\
\hline
\end{tabular}

Step 11. The ranking order of alternatives w.r.t. $\alpha_{j}$ is $\mathscr{A}_{1}>\mathscr{A}_{3}>\mathscr{A}_{2}$. The ranking order of alternatives w.r.t. $\beta_{j}$ is $\mathscr{A}_{1}>\mathscr{A}_{3}>\mathscr{A}_{2}$. The ranking order of alternatives w.r.t. $\gamma_{j}$ is $\mathscr{A}_{1}>\mathscr{A}_{3}>\mathscr{A}_{2}$.

All three ranking orders show that $\mathscr{A}_{1}$, i.e., mechanical recycling, is the best alternative. It is important to note that the ranking orders obtained by employing three different cosine similarity measures are the same which shows the effectiveness and compatibility of these cosine similarity measures. The three ranking orders are shown in Figure 6:

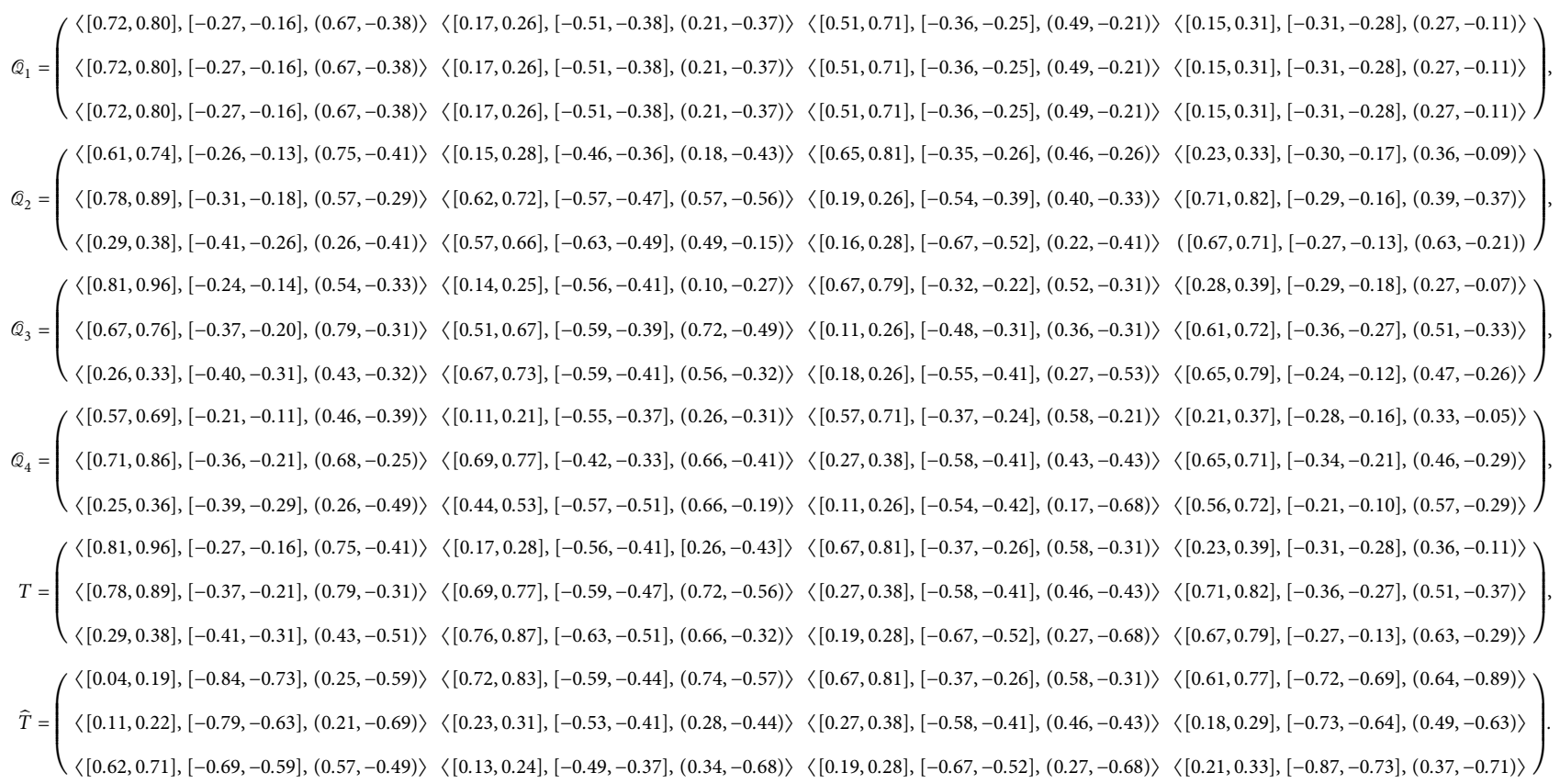

Table 5 shows the proposed CBF-TOPSIS method based on cosine SMs compared with some existing methods, and the ranking is summarized in Table 6. As shown in Table 6, the best alternative provided by any other technique acknowledges the validity and efficacy of the suggested MCGDM approach.

The existing MCGDM methods are designed to deal with vague information under some limitations imposed on membership grades. These methods cannot deal with cubic bipolar fuzzy information. The proposed mathematical models are more efficient and reliable to address bipolarity, vagueness, and fuzziness with cubic bipolar fuzzy sets. The computations provide a robust analysis for ranking of alternatives and the selection of feasible alternatives.

\section{Conclusion}

The researchers have developed different approaches, methods, models, and techniques to address vagueness and uncertainties in real-life problems. Bipolarity is a key factor in humanized computing that defines both positive and negative aspects in the objects. Bipolar fuzzy information with ordered pairs of positive and negatives grades and interval-valued bipolar fuzzy information with intervals of positive and negative grades are strong models to address bipolarity, respectively. A cubic bipolar fuzzy set (CBFS) is a new hybrid approach for dealing with vagueness, fuzziness, and bipolarity with BFS and IVBFS information simultaneously. The main objectives of the manuscript are itemized as follows: 
(1) Three different cosine SMs are developed for cubic bipolar fuzzy sets (CBFSs) based on the cosine of the angle between two vectors, new distance measures, and cosine function, respectively

(2) The problem of bacteria recognition is analyzed by using similarity measures for cubic bipolar fuzzy information

(3) An extended TOPSIS approach is developed with the help of similarity measures for MCGDM

(4) A practical application of the suggested MCDGM approach is presented towards sustainable plastic recycling process

A comparison analysis of the suggested technique with some existing techniques is also presented to depict the efficacy, validity, and superiority of the suggested technique [51].

\section{Data Availability}

Data sharing is not applicable to this article as no datasets were generated or analyzed during the current study.

\section{Conflicts of Interest}

The authors declare that they have no conflicts of interest.

\section{References}

[1] L. A. Zadeh, "Fuzzy sets," Information and Control, vol. 8, no. 3, pp. 338-353, 1965.

[2] L. A. Zadeh, "The concept of a linguistic variable and its application to approximate reasoning-I," Information Sciences, vol. 8, no. 3, pp. 199-249, 1975.

[3] K. T. Atanassov, "Intuitionistic fuzzy sets," Fuzzy Sets and Systems, vol. 20, no. 1, pp. 87-96, 1986.

[4] R. R. Yager, "Pythagorean fuzzy subsets," in Proceedings of the Joint IFSA World Congress and NAFIPS Annual Meeting, pp. 57-61, Edmonton, Canada, 2013.

[5] R. R. Yager, "Pythagorean membership grades in multicriteria decision making," IEEE Transactions on Fuzzy Systems, vol. 22, no. 4, pp. 958-965, 2014.

[6] R. R. Yager, "Generalized orthopair fuzzy sets," IEEE Transactions on Fuzzy Systems, vol. 25, no. 5, pp. 1220-1230, 2017.

[7] F. Smarandache, "A unifying field in logics: neutrosophic logic," Neutrosophy, Neutrosophic Set, Neutrosophic Probability and Statistics, Vol. 155, American Research Press, Washington D. C., USA, 4th edition, 2005.

[8] H. Wang, F. Smarandache, Y. Q. Zhang, and R. Sunderraman, "Single valued neutrosophic sets," Multispace and Multistructure, vol. 4, pp. 410-413, 2010.

[9] S. Ashraf and S. Abdullah, "Spherical aggregation operators and their application in multiattribute group decision-making," International Journal of Intelligent Systems, vol. 34, no. 3, pp. 493-523, 2019.

[10] T. Mahmood, K. Ullah, Q. Khan, and N. Jan, “An approach toward decision-making and medical diagnosis problems using the concept of spherical fuzzy sets," Neural Computing \& Applications, vol. 31, no. 11, pp. 7041-7053, 2019.
[11] F. K. Gundogdu and C. Kahraman, "Spherical fuzzy sets and spherical fuzzy TOPSIS method," Journal of Intelligent and Fuzzy Systems, vol. 36, no. 9-12, pp. 1-16, 2018.

[12] B. C. Cuong and V. Kreinovich, "Picture fuzzy sets-a new concept for computational intelligence problems," in Proceedings of the 2013 Third World Congress on Information and Communication Technologies (WICT 2013), pp. 1-6, Hanoi, Vietnam, December 2013.

[13] B. C. Cuong, "Picture fuzzy sets," Journal of Computer Science and Cybernetics, vol. 30, no. 4, pp. 409-420, 2014.

[14] W. R. Zhang, "Bipolar fuzzy sets and relations, a computational framework for cognitive modeling and multiagent decision analysis," in Proceedings of the 1994 IEEE Conference Fuzzy Information Processing Society Biannual Conference, pp. 305-309, San Antonio, TX, USA, December 1994.

[15] W. R. Zhang, "Bipolar fuzzy sets," in Proceedings of the IEEE Inrenational Conference on Fuzzy Systems, pp. 835-840, Anchorage, AK, USA, May 1998.

[16] K. M. Lee, "Bipolar-valued fuzzy sets and their basic operations," in Proceedings of the International Conference, pp. 307-317, Bangkok, Thailand, 2000.

[17] I. Deli, M. Ali, and F. Smarandache, "Bipolar neutrosophic sets and their application based on multi-criteria decision making problems," in Proceedings of the 2015 International Conference on Advanced Mechatronic Systems, Beijing, China, 2015.

[18] G. Wei, C. Wei, and H. Gao, "Multiple attribute decision making with interval-valued bipolar fuzzy information and their application to emerging technology commercialization evaluation," IEEE Access, vol. 6, pp. 60930-60955, 2018.

[19] Y. B. Jun, C. S. Kim, and K. O. Yang, "Cubic sets," Annals of Fuzzy Mathematics and Informatics, vol. 4, no. 1, pp. 83-98, 2012.

[20] J. Ye, "Cosine similarity measures for intuitionistic fuzzy sets and their applications," Mathematical and Computer Modelling, vol. 53, no. 1-2, pp. 91-97, 2011.

[21] G. Wei and Y. Wei, "Similarity measures of Pythagorean fuzzy sets based on the cosine function and their applications," International Journal of Intelligent Systems, vol. 33, no. 3, pp. 634-652, 2018.

[22] V. Uluçay, I. Deli, and M. Şahin, "Similarity measures of bipolar neutrosophic sets and their application to multiple criteria decision making," Neural Computing \& Applications, vol. 29, no. 3, pp. 739-748, 2018.

[23] M. Abdel-Basset, M. Mohamed, M. Elhoseny, L. H. Son, F. Chiclana, and A. E.-N. H. Zaied, "Cosine similarity measures of bipolar neutrosophic set for diagnosis of bipolar disorder diseases," Artificial Intelligence in Medicine, vol. 101, Article ID 101735, 2019.

[24] A. Tu, J. Ye, and B. Wang, "Multiple attribute decisionmaking method using similarity measures of neutrosophic cubic sets," Symmetry, vol. 10, no. 6, pp. 1-11, 2018.

[25] Z. Lu and J. Ye, "Cosine measures of neutrosophic cubic sets for multiple attribute decision-making," Symmetry, vol. 9, no. 7, p. 121, 2017.

[26] X. Peng, H. Yuan, and Y. Yang, "Pythagorean fuzzy information measures and their applications," International Journal of Intelligent Systems, vol. 32, no. 10, pp. 991-1029, 2017.

[27] X. Peng and L. Liu, "Information measures for q -rung orthopair fuzzy sets," International Journal of Intelligent Systems, vol. 34, no. 8, pp. 1795-1834, 2019.

[28] M. Riaz, K. Naeem, and D. Afzal, "A similarity measure under Pythagorean fuzzy soft environment with applications," 
Computational and Applied Mathematics, vol. 39, pp. 1-17, 2020.

[29] Z. Hussian and M. S. Yang, "Distance and similarity measures of Pythagorean fuzzy sets based on the Hausdorff metric with application to fuzzy TOPSIS," International Journal of Intelligent Systems, vol. 34, no. 10, pp. 2633-2654, 2019.

[30] C.-L. Hwang and K. Yoon, "Methods for multiple attribute decision making," in Multiple Attribute Decision Making, G. Fandel and W. Trockel, Eds., Springer, Berlin, Germany, pp. 58-191, 1981.

[31] X. Zhang and Z. Xu, "Extension of TOPSIS to multiple criteria decision making with Pythagorean fuzzy sets," International Journal of Intelligent Systems, vol. 29, no. 12, pp. 1061-1078, 2014.

[32] P. Rani, A. R. Mishra, G. Rezaei, H. Liao, and A. Mardani, "Extended Pythagorean fuzzy topsis method based on similarity measure for sustainable recycling partner selection," International Journal of Fuzzy Systems, vol. 22, no. 2, pp. 735-747, 2020.

[33] M. Akram, Shumaiza, and M. Arshad, "Bipolar fuzzy topsis and bipolar fuzzy electre-I methods to diagnosis," Journal of Computational and Applied Mathematics, vol. 39, pp. 1-21, 2019.

[34] H. Garg and R. Arora, "Topsis method based on correlation coefficient for solving decision-making problems with intuitionistic fuzzy soft set information," AIMS Mathematics, vol. 5, no. 4, pp. 2944-2966, 2020.

[35] H. Garg and G. Kaur, "Topsis based on nonlinear-programming methodology for solving decision-making problems under cubic intuitionistic fuzzy set environment," Computational and Applied Mathematics, vol. 38, pp. 1-19, 2019.

[36] M. Riaz and S. T. Tehrim, "Multi-attribute group decision making based on cubic bipolar fuzzy information using averaging aggregation operators," Journal of Intelligent \& Fuzzy Systems, vol. 37, no. 2, pp. 2473-2494, 2019.

[37] M. Riaz and S. T. Tehrim, "Cubic bipolar fuzzy ordered weighted geometric aggregation operators and their application using internal and external cubic bipolar fuzzy data," Computational and Applied Mathematics, vol. 38, no. 2, p. 87, 2019.

[38] M. Riaz and S. T. Tehrim, "Cubic bipolar fuzzy set with application to multi-criteria group decision making using geometric aggregation operators," Soft Computing, vol. 24, no. 16, pp. 16111-16133, 2020.

[39] Z. Ali, T. Mahmood, T. Mahmood, K. Ullah, and Q. Khan, "Einstein geometric aggregation operators using a novel complex interval-valued pythagorean fuzzy setting with application in green supplier chain management," Reports in Mechanical Engineering, vol. 2, no. 1, pp. 105-134, 2021.

[40] A. Alosta, O. Elmansuri, and I. Badi, "Resolving a location selection problem by means of an integrated AHP-RAFSI approach," Reports in Mechanical Engineering, vol. 2, no. 1, pp. 135-142, 2021.

[41] S. Hashemkhani Zolfani, M. Yazdani, D. Pamucar, and P. Zarate, "A VIKOR and TOPSIS focused reanalysis of the MADM methods based on logarithmic normalization," Facta Universitatis, Series: Mechanical Engineering, vol. 18, no. 3, pp. 341-355, 2020.

[42] K. R. Ramakrishnan and S. Chakraborty, "A cloud TOPSIS model for green supplier selection," Facta Universitatis-Series: Mechanical Engineering, vol. 18, no. 3, pp. 375-397, 2020.

[43] A. Dobrosavljevic and S. Urosevic, "Analysis of business process management defining and structuring activities in micro, small and medium sized enterprises," Operational Research in Engineering Sciences: Theory and Applications, vol. 2, no. 3, pp. 40-54, 2019.

[44] O. Yorulmaz, S. K. Yildirim, and B. F. Yildirim, "Robust Mahalanobis distance based TOPSIS to evaluate the economic development of provinces," Operational Research in Engineering Sciences: Theory and Applications, vol. 4, no. 2, pp. 102-123, 2021.

[45] I. Petrovic and M. Kankaras, "A hybridized IT2FS-DEMATEL-AHP-TOPSIS multicriteria decision making approach: case study of selection and evaluation of criteria for determination of air traffic control radar position," Decision Making: Applications in Management and Engineering, vol. 3, no. 1, pp. 146-164, 2020.

[46] I. Badi and D. Pamucar, "Supplier selection for steelmaking company by using combined grey-MARCOS methods," Decision Making: Applications in Management and Engineering, vol. 3, no. 2, pp. 37-48, 2020.

[47] M. Riaz, N. Cagman, N. Wali, and A. Mushtaq, "Certain Properties of Soft multi-set topology with applications in multi-criteria decision making," Decision Making: Applications in Management and Engineering, vol. 3, no. 2, pp. 70-96, 2020.

[48] Z. S. Mazhandu, E. Muzenda, T. A. Mamvura, M. Belaid, and T. Nhubu, "Integrated and consolidated review of plastic waste management and bio-based biodegradable plastics: challenges and opportunities," Sustainability, vol. 12, no. 20, p. $8360,2020$.

[49] W. d' Ambrieres, "Plastics recycling worldwide: current overview and desirable changes," Field Actions Science Reports, vol. 19, pp. 12-21, 2019.

[50] S. Eraslan and F. Karaaslan, "A group decision making method based on TOPSIS under fuzzy soft environment," Journal of New Theory, vol. 3, pp. 30-40, 2015.

[51] S. Geetha, S. Narayanamoorthy, J. V. Kureethara, D. Baleanu, and D. Kang, "The hesitant Pythagorean fuzzy ELECTRE III: an adaptable recycling method for plastic materials," Journal of Cleaner Production, vol. 291, no. 1, Article ID 125281, 2021. 